This item was submitted to Loughborough's Institutional Repository (https://dspace.lboro.ac.uk/) by the author and is made available under the following Creative Commons Licence conditions.

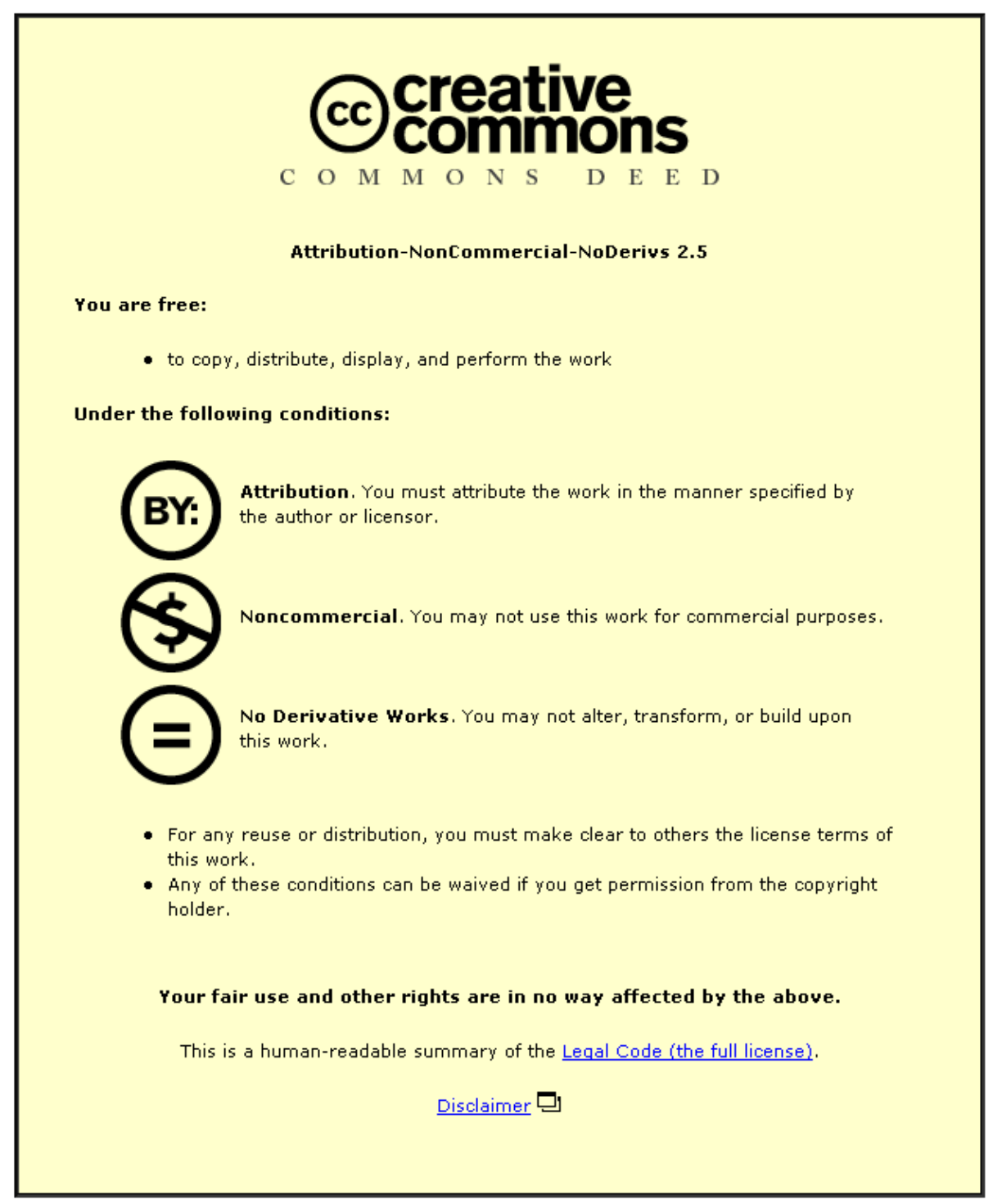

For the full text of this licence, please go to: http://creativecommons.org/licenses/by-nc-nd/2.5/ 


\title{
A UNIFIED CONSTRUCTION OF GENERALISED CLASSICAL POLYNOMIALS ASSOCIATED WITH OPERATORS OF CALOGERO-SUTHERLAND TYPE
}

\author{
MARTIN HALLNÄS AND EDWIN LANGMANN
}

\begin{abstract}
In this paper we consider a large class of many-variable polynomials which contains generalisations of the classical Hermite, Laguerre, Jacobi and Bessel polynomials as special cases, and which occur as the polynomial part in the eigenfunctions of Calogero-Sutherland type operators and their deformations recently found and studied by Chalykh, Feigin, Sergeev, and Veselov. We present a unified and explicit construction of all these polynomials.
\end{abstract}

\section{INTRODUCTION}

In this paper we discuss the construction of symmetric polynomials which arise as eigenfunctions of exactly solvable quantum many-body systems of CalogeroSutherland type $[6,51]$. In particular, we demonstrate that a particular construction method found and studied by us in simple special cases [20,30] can be naturally extended to the full class of such model. Our results provide a unified approach to many-variable generalisations of the classical Hermite, Lagueree, Jacobi and Bessel polynomials. We also show that it is natural to generalise our approach to a larger family of polynomials related to a deformation of the Calogero-Sutherland type systems found and studied by Chalykh, Feigin, Sergeev, and Veselov [7, 46, 47]. This allows us to derive, for each such polynomial, a family of different representations labeled by a pair of non-negative integers $(M, \tilde{M})$. Moreover, one can choose $(M, \tilde{M})$ such that the representation is simplest, i.e., a linear combination of certain explicitly given polynomials with the least number of terms. These results are nontrivial already for the non-deformed polynomials. To mention a specific example, we find that a Jack polynomial $[49]$ has a $(M, \tilde{M})$ representation if there exists a pair of non-negative integers $(m, \tilde{m})$ such that the Young diagram corresponding to this Jack polynomial can be covered by the union of the two rectangular Young diagrams of size $M \times m$ (i.e., $M$ rows and $m$ columns) and $\tilde{m} \times \tilde{M}$, respectively. Moreover, the simplest such representation is obtained by minimizing $M+\tilde{M}$. For example, the simplest possible representation of the Jack polynomial corresponding

Date: July 15, 2009.

1991 Mathematics Subject Classification. Primary 33C70; Secondary 81U15.

Key words and phrases. Calogero-Sutherland operators, many-variable polynomials, series representations, exactly solvable quantum many-body systems. 
to the Young diagrams

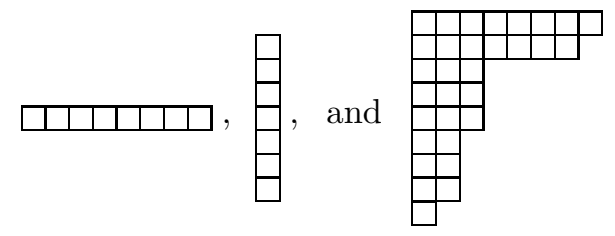

is for $(M, \tilde{M})=(1,0),(0,1)$, and $(2,3)$, respectively, and in the first two cases our representation actually consists only of a single term. The main tool in our construction are certain identities which, to each (deformed) Calogero-Sutherland system, relates a system of the same type. In special cases, these identities are well known and related to a duality of the Jack polynomials (see, e.g., [39]), or, more generally, the algebra homomorphism which maps Jack polynomials to so called super Jack polynomials (see, e.g., [48]). We deduce these identities in a unified manner, and thus unify, as well as extend, previously known results.

While we mention these examples in the beginning to give an impression of the kind of results we obtain, the emphasis in our presentation is on our construction method. To avoid overloading our formulae we explain this method in detail for the non-deformed polynomials and the representations $(M, 0)$. The subsequent extension to the general case is then remarkably easy.

1.1. Calogero-Sutherland type models. As mentioned above, the polynomials studied in this paper play an important role in physics since they occur in prominent exactly solvable quantum mechanical models defined by partial differential operators of Calogero-Sutherland type. These latter operators play a central role in our construction, and we therefore proceed to explain their relation to the classical Hermite, Laguerre, Jacobi and Bessel polynomials. As elaborated below, to each such sequence of one-variable polynomials $\left\{p_{n}: n=0,1, \ldots\right\}$ there exists a quantum mechanical model defined by a Schrödinger operator

$$
h=-\partial_{x}^{2}+V(x),
$$

with a particular potential function $V$ such that its eigenfunctions are of the form

$$
\psi_{n}(x)=\psi_{0}(x) p_{n}(z) \text { with } z=z(x)
$$

for particular $C^{2}$ functions $\psi_{0}(x)$ and $z(x)$ ( $x$ is a real variable, and we use the notation $\left.\partial_{x}:=\frac{\partial}{\partial x}\right)$. Many-variable generalisation of these polynomials appear in the following generalisation to an arbitrary number $N$ of particles: each one-particle Schrödinger operator (1) allows for a many-body generalisation

$$
H_{N}=\sum_{j=1}^{N}\left(-\partial_{x_{j}}^{2}+V\left(x_{j}\right)\right)+\kappa(\kappa-1) \sum_{j<k} W\left(x_{j}, x_{k}\right)
$$

with $W$ a particular two-body interaction potential such that $H_{N}$ has eigenfunctions $\Psi_{\mathbf{n}}$ which are labelled by integer vectors $\mathbf{n}=\left(n_{1}, \ldots, n_{N}\right)$ and are of the form

$$
\begin{gathered}
\Psi_{\mathbf{n}}(\mathbf{x})=\Psi_{0}(\mathbf{x}) P_{\mathbf{n}}(\mathbf{z}) \text { with } z_{j}=z\left(x_{j}\right), \\
\Psi_{0}(\mathbf{x})=\prod_{j=1}^{N} \psi_{0}\left(x_{j}\right) \prod_{j<k}\left(z_{k}-z_{j}\right)^{\kappa}
\end{gathered}
$$


where the functions $P_{\mathbf{n}}$ are certain symmetric polynomials in $N$ variables $\mathbf{z}=$ $\left(z_{1}, \ldots, z_{N}\right)$ generalising the polynomials $p_{n}$. To avoid certain technicalities and since it includes the cases of main physical interests we will assume $\kappa>0$, even though many of our results actually hold true also for other values of $\kappa$. We mention already at this point that although the symmetry of the polynomials $P_{\mathbf{n}}$ implies that a complete set of eigenfunctions can be labelled by partitions, i.e., sequences $\mathbf{n}=\boldsymbol{\lambda}=\left(\lambda_{1}, \ldots, \lambda_{N}\right)$ of non-negative integers $\lambda_{j}$ such that $\lambda_{1} \geq \cdots \geq \lambda_{N}$, it is in our approach not always natural to make this restriction, as will become evident below. These operators $H_{N}$ define exactly solvable quantum-many body systems of Calogero-Sutherland type $[6,22,42,50]$; see Table 1 for a list of well-known examples (note that we have added constants to the potentials $V$ to simplify certain formulae later on).

1.2. Deformed Calogero-Sutherland operators. A further interesting generalisation of the one-particle Schrödinger operators (10) is the following class of differential operators in two sets of variables $\mathbf{x}=\left(x_{1}, \ldots, x_{N}\right)$ and $\tilde{\mathbf{x}}=\left(\tilde{x}_{1}, \ldots, \tilde{x}_{\tilde{N}}\right)$, where $N$ and $\tilde{N}$ are arbitrary non-negative integers:

$$
\begin{aligned}
& H_{N, \tilde{N}}=\sum_{j=1}^{N}(\left.-\partial_{x_{j}}^{2}+V\left(x_{j}\right)\right)-\sum_{J=1}^{\tilde{N}} \kappa\left(-\partial_{\tilde{x}_{J}}^{2}+\tilde{V}\left(\tilde{x}_{J}\right)\right) \\
&+\kappa(\kappa-1) \sum_{j<k} W\left(x_{j}, x_{k}\right)+\frac{\kappa-1}{\kappa} \sum_{J<K} W\left(\tilde{x}_{j}, \tilde{x}_{k}\right) \\
&+(1-\kappa) \sum_{j, K} W\left(x_{j}, \tilde{x}_{K}\right)
\end{aligned}
$$

with a potential function $\tilde{V}$ of the same kind as $V$ but with different parameters, as specified in Table 1. These differential operators are natural generalisations of the Schrödinger operator (3) in that they also have polynomial eigenfunctions with corresponding eigenvalues that can be computed explicitly; see Section 4 for a precise formulation of this result. In this paper we present an explicit construction of all these many-variable generalisations of the above mentioned classical polynomials. We obtain the results by a unified treatment making no reference to special cases, as explained below.

1.3. Construction method. To explain the nature of our results we now discuss the standard Calogero-Sutherland cases $\tilde{N}=0$ in more detail. Our approach is based on a set of remarkable identities which provide us with particular sets of symmetric polynomials on which the action of the differential operators (3) is particularly simple. These latter polynomials can be defined by the following expansion:

$$
\frac{\prod_{1 \leq j<k \leq N}\left(1-w_{j} / w_{k}\right)^{\kappa}}{\prod_{j, k=1}^{N}\left(1-z_{j} / w_{k}\right)^{\kappa}}=\sum_{\mathbf{n} \in \mathbb{Z}^{N}} f_{\mathbf{n}}(\mathbf{z}) w_{1}^{-n_{1}} w_{2}^{-n_{2}} \cdots w_{N}^{-n_{N}}
$$

valid for $\left|w_{N}\right|>\left|w_{N-1}\right|>\cdots>\left|w_{1}\right|>\max _{k}\left(\left|z_{k}\right|\right)$. We mention that these polynomials were first defined in [30] by a certain contour integral. However, using Cauchy's theorem it is easy to verify that the two definitions are equivalent. We explicitly construct series representations for the polynomials $P_{\mathbf{n}}$ in (4) which are of the form $P_{\mathbf{n}}(\mathbf{z})=\sum_{\mathbf{m}} u_{\mathbf{n}}(\mathbf{m}) f_{\mathbf{n}}(\mathbf{z})$ and, in addition, we obtain certain completeness 
results; see Section 3 for the precise statements. We will also derive a partial generalisation of these results to the differential operators $H_{N, \tilde{N}}$; see Section 4 . We thus generalise, unify, and extend various results which were known before only in special cases $[20,31]$.

We now explain in which sense our approach is unified and give a precise definition of the classical polynomials we consider: each such sequence consists of polynomials $p_{n}$ of order $n=0,1, \ldots$ such that they are common eigenfunctions of a differential operator

$$
\tilde{h}=-\psi_{0}^{-1} h \psi_{0}=\alpha(z) \partial_{z}^{2}+\beta(z) \partial_{z}
$$

with

$$
\alpha(z)=\alpha_{2} z^{2}+\alpha_{1} z+\alpha_{0} \text { and } \beta(z)=\beta_{1} z+\beta_{0}
$$

for some real coefficients $\alpha_{j}$ and $\beta_{j}$; see Table 2 for the polynomials corresponding to our examples in Table 1. It is interesting to note that the Schrödinger operator $h$ in (1) with the eigenfunctions (2) can be fully characterised by these polynomials $\alpha$ and $\beta$ : it is straightforward to verify that

\begin{tabular}{|c|c|c|c|}
\hline & $V(x)$ & $W(x, y)$ & $\tilde{V}(x)$ \\
\hline I & $\omega^{2} x^{2}-\omega$ & $\frac{2}{(x-y)^{2}}$ & $\tilde{\omega}^{2} x^{2}-\tilde{\omega}$ \\
\hline II & 0 & $\frac{1}{2 \sin ^{2}\left(\frac{x-y}{2}\right)}$ & $\left(\kappa^{2}-1\right) / \kappa^{4}$ \\
\hline III & $-\frac{c(c+1)}{\cosh ^{2}(x)}+c^{2}$ & $\frac{1}{2 \sinh ^{2}\left(\frac{x-y}{2}\right)}-\frac{1}{2 \cosh ^{2}\left(\frac{x+y}{2}\right)}$ & $-\frac{\tilde{c}(\tilde{c}+1)}{\cosh ^{2}(x)}+\tilde{c}^{2}$ \\
\hline IV & $\begin{array}{c}\omega^{2} x^{2}+\frac{a(a-1)}{x^{2}} \\
-\omega(1+2 a)\end{array}$ & $\frac{2}{(x-y)^{2}}+\frac{2}{(x+y)^{2}}$ & $\begin{array}{c}\tilde{\omega}^{2} x^{2}+\frac{\tilde{a}(\tilde{a}-1)}{x^{2}} \\
-\tilde{\omega}(1+2 \tilde{a})\end{array}$ \\
\hline V & $\frac{a(a-1)}{\sin ^{2} x}-a^{2}$ & $\frac{1}{2 \sin ^{2}\left(\frac{x-y}{2}\right)}+\frac{1}{2 \sin ^{2}\left(\frac{x+y}{2}\right)}$ & $\frac{\tilde{a}(\tilde{a}-1)}{\sin ^{2} x}-\tilde{a}^{2}$ \\
\hline VI & $\begin{array}{c}\frac{a(a-1)}{4 \sin ^{2}\left(\frac{x}{2}\right)}+\frac{b(b-1)}{4 \cos ^{2}\left(\frac{x}{2}\right)} \\
-\frac{(a+b)^{2}}{4}\end{array}$ & $\frac{1}{2 \sin ^{2}\left(\frac{x-y}{2}\right)}+\frac{1}{2 \sin ^{2}\left(\frac{x+y}{2}\right)}$ & $\begin{array}{c}\frac{\tilde{a}(\tilde{a}-1)}{4 \sin ^{2}\left(\frac{x}{2}\right)}+\frac{\tilde{b}(\tilde{b}-1)}{4 \cos ^{2}\left(\frac{x}{2}\right)} \\
-\frac{(\tilde{a}+\tilde{b})^{2}}{4}\end{array}$ \\
\hline VII & $\begin{array}{c}\omega^{2} e^{-2 x} \\
-\omega(1+2 c) e^{-x}+c^{2}\end{array}$ & $\frac{1}{2 \sinh ^{2}\left(\frac{x-y}{2}\right)}$ & $\begin{array}{c}\tilde{\omega}^{2} e^{-2 x} \\
-\tilde{\omega}(1+2 \tilde{c}) e^{-x}+\tilde{c}^{2}\end{array}$ \\
\hline
\end{tabular}

(10) $V(x)=v(z(x)), \quad v(z)=\frac{\left(2 \beta(z)-\alpha^{\prime}(z)\right)\left(2 \beta(z)-3 \alpha^{\prime}(z)\right)}{16 \alpha(z)}-\frac{1}{4} \alpha^{\prime \prime}(z)+\frac{1}{2} \beta^{\prime}(z)$

TABLE 1. Examples of external- and two-body interaction potentials such that the differential operators (3) and (6) have polynomial eigenfunctions. The parameters in the last column are: $\tilde{\omega}=-\omega / \kappa, \tilde{c}=-(2 c+\kappa+1) /(2 \kappa), \tilde{a}=-(2 a-\kappa-1) /(2 \kappa)$ and $\tilde{b}=-(2 b-\kappa-1) /(2 \kappa)$. 
(the prime here and in the following indicates differentiation) with $z(x)$ a solution of the differential equation

$$
z^{\prime}(x)^{2}=\alpha(z(x))
$$

and

$$
\psi_{0}(x)=e^{-w(z(x))}
$$

with $w(z)$ any solution of the differential equation

$$
w^{\prime}(z)=\frac{\alpha(z)^{\prime}-2 \beta(z)}{4 \alpha(z)} .
$$

Moreover, the potential functions $W$, ensuring that the differential operators $H_{N}$ in (3) and $H_{N, \tilde{N}}$ in (6) have polynomial eigenfunctions, are given by

$$
W\left(x_{1}, x_{2}\right)=\frac{\alpha\left(z_{1}\right)+\alpha\left(z_{2}\right)}{\left(z_{1}-z_{2}\right)^{2}}-\alpha_{2} \text { with } z_{j}=z\left(x_{j}\right) .
$$

The constant $\alpha_{2}$ is subtracted to simplify formulae later on, and $\tilde{V}$ is given by a formula as in (10) but with $\beta(z)$ replaced by

$$
\tilde{\beta}(z)=\left[(1+\kappa) \alpha^{\prime}(z)-\beta(z)\right] / \kappa .
$$

It is interesting to note that the exact eigenvalues of the differential operators $H_{N}$ in (3) and $H_{N, \tilde{N}}$ in (6) are determined by the leading coefficients of these polynomials $\alpha(z)$ and $\beta(z)$. For example, the exact eigenvalues of $H_{N}$ corresponding to the eigenfunctions (4) are

$$
E_{\mathbf{n}}=E_{0}-\sum_{j=1}^{N}\left(\alpha_{2} n_{j}\left(n_{j}-1\right)+\left(2 \kappa \alpha_{2}(N-j)+\beta_{1}\right) n_{j}\right)
$$

with

$$
E_{0}=-\frac{\alpha_{2} \kappa^{2}}{3} N\left(N^{2}-1\right)-\frac{\kappa\left(\beta_{1}-(1+\kappa) \alpha_{2}\right)}{2} N(N-1) .
$$

This result, well-known in many special cases (see, e.g., [14]), will naturally emerge from our construction in Section 3 We are now in a position to state more precisely in which sense our approach is unified: in our construction information concerning the different cases will enter only via the two polynomials $\alpha$ and $\beta$. We also stress that the special cases listed in Table 2 are only intended as examples, and that our approach is for arbitrary polynomials $\alpha$ and $\beta$ of the form (9).

1.4. Special cases. To put this general scheme into perspective we now discuss its well-known special cases summarised in Tables 1 and 2. The case $\alpha=1$ and $\beta(z)=-2 \omega z$ corresponds to the exactly solvable many-body generalisation of the quantum harmonic oscillator introduced by Calogero [6] (case I in Tables 1 and 2), while the Sutherland model [50,51], generalising the quantum model of a free particle on a circle, is obtained by setting $\alpha(z)=-z^{2}$ and $\beta(z)=-z$ (case II). These models correspond to the Hermite polynomials and the ordinary monomials $p_{n}(z)=z^{n}$, respectively. The many-body models associated with the Legendre, Gegenbauer and Jacobi polynomials (cases IV, V and VI) are identical with Olshanetsky and Perelomov's $B_{N^{-}}$and $B C_{N^{-}}$variants of the Calogero- and Sutherland models; see [42] and references therein. An exact solution to the many-body generalisation of the Morse potential (case VII), corresponding to the generalised 


\begin{tabular}{|c|c|c|c|c|c|}
\hline & $\psi_{0}(x)$ & $z(x)$ & $p_{n}(z)$ & $\alpha(z)$ & $\beta(z)$ \\
\hline I & $e^{-\omega x^{2} / 2}$ & $x$ & $\begin{array}{l}H_{n}(\sqrt{\omega} z) \\
\text { (Hermite) }\end{array}$ & 1 & $-2 \omega z$ \\
\hline II & 1 & $e^{i x}$ & $z^{n}$ & $-z^{2}$ & $-z$ \\
\hline III & $\cosh ^{-c}(x)$ & $i \sinh (x)$ & $\begin{array}{c}C_{n}^{(-c)}(z) \\
\text { (Gegenbauer) }\end{array}$ & $-1+z^{2}$ & $(1-2 c) z$ \\
\hline IV & $e^{-\omega x^{2} / 2} x^{a}$ & $x^{2}$ & $\begin{array}{l}L_{n}^{\left(a-\frac{1}{2}\right)}(\omega z) \\
\text { (Laguerre) }\end{array}$ & $4 z$ & $2+4 a-4 \omega z$ \\
\hline $\mathrm{V}$ & $\sin ^{a}(x)$ & $\cos (x)$ & $\begin{array}{c}C_{n}^{(a)}(z) \\
\text { (Gegenbauer) }\end{array}$ & $1-z^{2}$ & $-(1+2 a) z$ \\
\hline VI & $\sin ^{a}\left(\frac{x}{2}\right) \cos ^{b}\left(\frac{x}{2}\right)$ & $\cos (x)$ & $\begin{array}{l}P_{n}^{\left(a-\frac{1}{2}, b-\frac{1}{2}\right)}(z) \\
\quad(\text { Jacobi })\end{array}$ & $1-z^{2}$ & $\begin{array}{c}b-a- \\
(1+a+b) z\end{array}$ \\
\hline VII & $\exp \left(-\omega e^{-x}-c x\right)$ & $e^{x}$ & $\begin{array}{c}y_{n}(z, 1-2 c, 2 \omega) \\
\quad(\text { gen. Bessel) }\end{array}$ & $z^{2}$ & $2 \omega+(1-2 c) z$ \\
\hline
\end{tabular}

TABLE 2. Exact solutions of the one-body Schrödinger equation associated with classical polynomials, as described in (1) and (2). Given are also the associated polynomials $\alpha$ and $\beta$; see (8)-(12). More details about the polynomials $p_{n}$ can be found in [1] (cases I and III-VI) and in [16] (case VII).

Bessel polynomials [26], was first given by Inozemtsev and Meshcheryakov [22]. A further study of this case can be found in [19]. There exist various other interesting special cases which we have not explored in detail, but they can all be transformed to the cases mentioned above by a rescaling and a translation of the variable $z$. The deformations of the Calogero-Sutherland models defined by $H_{N, \tilde{N}}$ in (6) were found and explored by Chalykh, Feigin, Sergeev, and Veselov; see, e.g., [7,46-48]. We will refer to these differential operators $H_{N, \tilde{N}}$ as deformed Calogero-Sutherland operators.

1.5. Related previous work. We now discuss the relation of our results to previous work in the literature. The many-variable polynomials corresponding to the eigenfunctions of the Calogero-Sutherland type models in cases I-VI in Table 1 have been extensively studied in the mathematics literature; see, e.g., $[11,39]$ and references therein. We mention, in particular, Heckman and Opdam's root system generalisation of the Jacobi polynomials [21] and the work of Baker and Forrester [3], van Diejen [9], and Lassalle [33-35], as well as of Macdonald [38], on many-variable generalisations of the classical orthogonal Hermite, Laguerre and Jacobi polynomials. A particularly well-studied case are the so-called Jack polynomials corresponding to the Sutherland model (case II). Explicit formulae for the Jack polynomials were recently obtained by Lassalle and Schlosser [37] (see 
also [36]) by inverting a so-called Pieri formula. For very particular values of the integer vector $\mathbf{n}=\left(n_{1}, \ldots, n_{N}\right)$ or a low number of variables explicit series expansions of the Jack polynomials were obtained by Stanley [49]. In addition, there exist expansions of the Jack, as well as certain other many-variable classical orthogonal polynomials, which are of a combinatorial nature $[8,10,27,39]$. These combinatorial results also include a formula resulting from the use of Sutherland's original solution algorithm [51]. We also mention operator solutions of the Calogero- and Sutherland models obtained in $[5,24,25,52,53]$ and [32], respectively, as well as integral representations of the Jack polynomials; see $[2,40,41]$ and references therein. Our results in this paper provide an explicit construction of the many-variable polynomials $P_{\mathbf{n}}$. These results were recently announced in [17], and they generalise those previously obtained by us for the Sutherland- [30] and the Calogero models [20]. We mention that our unified treatment of all the cases in Table 1 is different from the one based on root systems (see, e.g., [42]), and that it has been previously used by Gomez-Ullate et.al. [14] to obtain the energy eigenvalues in (14) and (15) by purely algebraic means. We also mention that our point of view is closely related to the theory of quasi-exactly solvable Schrödinger operators; see, e.g., $[4,15]$. Moreover, the identities which are the key to our results are stated in Corollaries 2.2 and 2.3 below, and they were known before only in special cases: an important special case of these identities for the Sutherland model (case II) is a consequence of a well-known result on Jack polynomials which, to our knowledge, is due to Stanley (see Proposition 2.1 in [49]), and a generalisation of the latter to the deformed case and other non-deformed cases can be found in [48] and [13,39,45], respectively. These identities relate Schrödinger- or deformed Calogero-Sutherland operators with different parameter values and are natural quantum analogs of the Bäcklund transformations for the classical Calogero-Moser models first found by Woijekowski [54], as discussed by Kutznetsov and Sklyanin [29]. We obtain these identities as specialisations of a particular identity stated in Proposition 2.1 which has the natural physical interpretation of giving the groundstate of a generalisation of the Schrödinger operator (3) where the particles are allowed to have different masses $m_{j}$ and with specific mass dependent external potentials $V_{m_{j}}\left(x_{j}\right)$. This is a powerful result of independent interest, and to our knowledge it was previously known only in the special cases I, II and IV in Table 11 [12, 20,44]. We finally mention an integral representation of the Jack polynomials recently obtained by Kuznetsov et.al. [28] using a separation-of-variables approach which is also based on the identity in Corollary 2.2.

1.6. Plan of the paper. In Section 2 we derive and discuss the identities which are the key to our solution method. Section 3 contains our explicit construction of series representation of all many-variable polynomials $P_{\mathbf{n}}$ determining the eigenfunctions (4) of the Schrödinger operators (3). In Section 4 we extend our construction of polynomial eigenfunctions to the deformed Calogero-Sutherland operators (66). We conclude with a few remarks in Section 5 .

1.7. Notation. We denote by $\mathbb{Z}, \mathbb{N}, \mathbb{N}_{0}$ and $\mathbb{R}$ the sets of all integers, positive integers, non-negative integers and real numbers, respectively. We shall say that an integer vector $\mathbf{n}=\left(n_{1}, n_{2}, \ldots\right)$ is of length $N$, denoted $\ell(\mathbf{n})=N$, if $N$ is the smallest non-negative integer such that the parts $n_{j}$ of $\mathbf{n}$ are zero for $j>N$. The symbols $\mathbf{n}$ and $\mathbf{m}$ will in most instances denote integer vectors, except in Section 2 
where the symbols $m_{j}$ are used for real 'mass parameters' rather than for parts of an integer vector $\mathbf{m}$. We will use the symbols $\boldsymbol{\lambda}, \boldsymbol{\mu}$ and $\boldsymbol{\nu}$ to emphasise that a particular integer vector is a partition. We will also use the notation

$$
\mathbf{x}^{\mathbf{s}}=x_{1}^{s_{1}} \cdots x_{N}^{s_{N}}
$$

where $\mathbf{x}=\left(x_{1}, \ldots, x_{N}\right)$ and $\mathbf{s}=\left(s_{1}, \ldots, s_{N}\right)$. Moreover, for $\mathbf{m} \in \mathbb{Z}^{M}$ and $\mathbf{n} \in \mathbb{Z}^{N}$ we will write $(\mathbf{m}, \mathbf{n})$ short for $\left(m_{1}, \ldots, m_{M}, n_{1}, \ldots, n_{N}\right)$.

\section{IDENTITIES}

In this section we present and prove a particular identity associated with the one-particle Schrödinger operator (11). The identities underlying our construction of eigenfunctions of the differential operators in (3) and (6) are stated in Section 2.2 and obtained as special cases of this more general results. Throughout the section, we assume the polynomials $\alpha$ and $\beta$ in (9) fixed, and $z(x)$ and $W\left(x_{1}, x_{2}\right)$ are as in (11) and (13).

2.1. Source identity. The following identity can be interpreted as providing the exact groundstate of a generalisation of the Schrödinger operator (3) where the particles can have different masses $m_{j}$. However, we will allow these parameters $m_{j}$ also to be negative, and we will use this identity as a source from which we obtain various other identities as special cases. To state this result we find it convenient to denote this generalised Schrödinger operators as $\mathcal{H}$, the particle coordinates as $X_{j}$, and the particle number as $\mathcal{N}$.

Proposition 2.1. Let

$$
\mathcal{H}=\sum_{j=1}^{\mathcal{N}} \frac{1}{m_{j}}\left(-\partial_{X_{j}}^{2}+V_{m_{j}}\left(X_{j}\right)\right)+\sum_{j<k} \frac{\kappa}{2}\left(\kappa m_{j} m_{k}-1\right)\left(m_{j}+m_{k}\right) W\left(X_{j}, X_{k}\right)
$$

with $m_{j}$ arbitrary real and non-zero parameters and

$$
V_{m}(X)=v_{m}(z(X))
$$

with

$$
v_{m}(z)=\frac{\left(2 \beta_{m}(z)-\alpha^{\prime}(z)\right)\left(2 \beta_{m}(z)-3 \alpha^{\prime}(z)\right)}{16 \alpha(z)}-\frac{1}{4} \alpha^{\prime \prime}(z)+\frac{1}{2} \beta_{m}^{\prime}(z),
$$

where the prime indicates differentiation with respect to the argument $z$ and

$$
\beta_{m}(z)=m \beta(z)+\frac{1}{2}(1-m)(1-\kappa m) \alpha^{\prime}(z) .
$$

Furthermore, let

$$
\Phi_{0}(\mathbf{X})=\prod_{j=1}^{\mathcal{N}} \psi_{0, m_{j}}\left(X_{j}\right) \prod_{j<k}\left(Z_{k}-Z_{j}\right)^{\kappa m_{j} m_{k}}
$$

with $Z_{j}=z\left(X_{j}\right)$ and

$$
\psi_{0, m}(X)=e^{-w_{m}(z(X))}
$$

with $w_{m}(z)$ any solution of the differential equation

$$
w_{m}^{\prime}(z)=\frac{\alpha^{\prime}(z)-2 \beta_{m}(z)}{4 \alpha(z)} .
$$


Then

$$
\left(\mathcal{H}-\mathcal{E}_{0}\right) \Phi_{0}=0
$$

with the constant

$$
\mathcal{E}_{0}=-\frac{g^{2} \alpha_{2}}{3}\left(|\mathbf{m}|^{3}-\left|\mathbf{m}^{3}\right|\right)-\frac{\kappa\left(\beta_{1}-(1+\kappa) \alpha_{2}\right)}{2}\left(|\mathbf{m}|^{2}-\left|\mathbf{m}^{2}\right|\right),
$$

where

$$
\left|\mathbf{m}^{n}\right|:=\sum_{j=1}^{\mathcal{N}} m_{j}^{n} \text { for } n=1,2,3 .
$$

The $m$-dependence of the external potentials and one-particle groundstate eigenfunctions for our examples is given in Table 3 with $m$-dependent parameters defined in the table captions.

Remark 2.1. Note that $\beta_{1}$ and $\beta_{0}$ always refers to the coefficients of the polynomial $\beta(z)$ as defined in (9), and are not to be confused with $\beta_{m}(z)$ for $m=1$ and $m=0$. There should be no danger of confusion since we always write $\beta(z)$ for $\beta_{m}(z)$ if $m=1$ and assume $m \neq 0$.

\begin{tabular}{|c|c|c|}
\hline & $V_{m}(x)$ & $\psi_{0, m}(x)$ \\
\hline I & $\omega_{m}^{2} x^{2}-\omega_{m}$ & $e^{-\omega_{m} x^{2} / 2}$ \\
II & $-\frac{1}{4} \kappa^{2} m^{2}(m-1)^{2}$ & $e^{i \kappa m(m-1) x / 2}$ \\
III & $-\frac{c_{m}\left(c_{m}+1\right)}{\cosh ^{2}(x)}+c_{m}^{2}$ & $\cosh ^{-c_{m}}(x)$ \\
IV & $\omega_{m}^{2} x^{2}+\frac{a_{m}\left(a_{m}-1\right)}{x^{2}}-\omega_{m}\left(1+2 a_{m}\right)$ & $e^{-\omega_{m} x^{2} / 2} x^{a_{m}}$ \\
V & $\frac{a_{m}\left(a_{m}-1\right)}{\sin ^{2} x}-a_{m}^{2}$ & $\sin ^{a_{m}}(x)$ \\
VI & $\frac{a_{m}\left(a_{m}-1\right)}{4 \sin ^{2}\left(\frac{x}{2}\right)}+\frac{b_{m}\left(b_{m}-1\right)}{4 \cos ^{2}\left(\frac{x}{2}\right)}-\frac{1}{4}\left(a_{m}+b_{m}\right)^{2}$ & $\sin ^{a_{m}}\left(\frac{x}{2}\right) \cos ^{b_{m}}\left(\frac{x}{2}\right)$ \\
VII & $\omega_{m}^{2} e^{-2 x}-\omega_{m}\left(1+2 c_{m}\right) e^{-x}+c_{m}^{2}$ & $e^{-\omega_{m} e^{-x}-c_{m} x}$ \\
\hline
\end{tabular}

TABLE 3. 'Mass' dependence of the external potentials and oneparticle groundstates in our examples, according to Proposition 2.1. The $m$-dependence of the parameters is as follows, $\omega_{m}=m \omega, c_{m}=m c-\frac{1}{2} \kappa m(m-1), a_{m}=m a+\frac{1}{2} \kappa m(m-1)$, and $b_{m}=m b+\frac{1}{2} \kappa m(m-1)$.

Proof of Proposition 2.1. We will show by straightforward computations that

$$
\mathcal{H}=\sum_{j=1}^{\mathcal{N}} \frac{1}{m_{j}} Q_{j}^{+} Q_{j}^{-}+\mathcal{E}_{0},
$$

where

$$
Q_{j}^{ \pm}=\mp \partial_{X_{j}}+\mathcal{V}_{j}, \quad \mathcal{V}_{j}(\mathbf{x})=\frac{1}{\Phi_{0}(\mathbf{x})} \partial_{X_{j}} \Phi_{0}(\mathbf{x})
$$


Since $Q_{j}^{-} \Phi_{0}=0$ for all $j$, this will prove the identity in (20).

To prove (23) we compute

$$
\mathcal{V}_{j}(\mathbf{x})=-w_{m_{j}}^{\prime}\left(z\left(X_{j}\right)\right) z^{\prime}\left(X_{j}\right)+\sum_{k \neq j} \kappa m_{j} m_{k} \frac{z^{\prime}\left(X_{j}\right)}{z\left(X_{j}\right)-z\left(X_{k}\right)},
$$

and thus

$$
\sum_{j=1}^{\mathcal{N}} \frac{1}{m_{j}} Q_{j}^{+} Q_{j}^{-}=-\sum_{j=1}^{\mathcal{N}} \frac{1}{m_{j}} \partial_{X_{j}}^{2}+\mathcal{W}_{1}+\mathcal{W}_{2}+\mathcal{W}_{3}
$$

with

$$
\mathcal{W}_{1}=\sum_{j=1}^{\mathcal{N}} \frac{1}{m_{j}}\left(w_{m_{j}}^{\prime}\left(Z_{j}\right)^{2} \alpha\left(Z_{j}\right)-w_{m_{j}}^{\prime \prime}\left(Z_{j}\right) \alpha\left(Z_{j}\right)-\frac{1}{2} w_{m_{j}}^{\prime}\left(Z_{j}\right) \alpha^{\prime}\left(Z_{j}\right)\right)
$$

the one-body terms,

$$
\mathcal{W}_{2}=\sum_{j=1}^{\mathcal{N}} \sum_{k \neq j} \kappa m_{k}\left(\left(\kappa m_{j} m_{k}-1\right) \frac{\alpha\left(Z_{j}\right)}{\left(Z_{j}-Z_{k}\right)^{2}}+\frac{\frac{1}{2} \alpha^{\prime}\left(Z_{j}\right)-2 w_{m_{j}}^{\prime}\left(Z_{j}\right) \alpha\left(Z_{j}\right)}{Z_{j}-Z_{k}}\right)
$$

the two-body terms, and

$$
\mathcal{W}_{3}=\sum_{j=1}^{\mathcal{N}} \sum_{k, \ell \neq j, k \neq \ell} \kappa^{2} m_{j} m_{k} m_{\ell} \frac{\alpha\left(Z_{j}\right)}{\left(Z_{j}-Z_{k}\right)\left(Z_{j}-Z_{\ell}\right)}
$$

the three-body terms; we inserted the relations $z^{\prime}\left(X_{j}\right)^{2}=\alpha\left(Z_{j}\right)$ and $z^{\prime \prime}\left(X_{j}\right)=$ $\frac{1}{2} \alpha^{\prime}\left(Z_{j}\right)$ and used the short hand notation $z\left(X_{j}\right)=Z_{j}$.

Symmetrising the sum defining $\mathcal{W}_{3}$ and inserting $\alpha\left(Z_{j}\right)=\sum_{p=0}^{2} \alpha_{p} Z_{j}^{p}$ we can write

$$
\mathcal{W}_{3}=\sum_{j<k<\ell} 2 \kappa^{2} m_{j} m_{k} m_{\ell} \sum_{p=0}^{2} \alpha_{p} \frac{Z_{j}^{p}\left(Z_{k}-Z_{\ell}\right)-Z_{k}^{p}\left(Z_{j}-Z_{\ell}\right)+Z_{\ell}^{p}\left(Z_{j}-Z_{k}\right)}{\left(Z_{j}-Z_{k}\right)\left(Z_{j}-Z_{\ell}\right)\left(Z_{k}-Z_{\ell}\right)}
$$

and observing that the fraction on the r.h.s. is identical with 0 and 1 for $p=0,1$ and $p=2$, respectively, we find that $\mathcal{W}_{3}$ is equal to the constant

$$
\mathcal{W}_{3}=\sum_{j<k<\ell} 2 \kappa^{2} m_{j} m_{k} m_{\ell} \alpha_{2}
$$

Symmetrising the sum defining $\mathcal{W}_{2}$ and inserting $\frac{1}{2} \alpha^{\prime}\left(Z_{j}\right)-2 w_{m_{j}}^{\prime}\left(Z_{j}\right) \alpha\left(Z_{j}\right)=$ $\beta_{m_{j}}\left(Z_{j}\right)$, following from the second equation in (19), we obtain

$$
\mathcal{W}_{2}=\sum_{j<k} \kappa\left(\left(\kappa m_{j} m_{k}-1\right) \frac{m_{k} \alpha\left(Z_{j}\right)+m_{j} \alpha\left(Z_{k}\right)}{\left(Z_{j}-Z_{k}\right)^{2}}+\frac{m_{k} \beta_{m_{j}}\left(Z_{j}\right)-m_{j} \beta_{m_{k}}\left(Z_{k}\right)}{Z_{j}-Z_{k}}\right) .
$$

We now decompose $\mathcal{W}_{2}$ into two parts as follows:

$$
\mathcal{W}_{2}=\mathcal{W}_{2,0}+\mathcal{W}_{2,1}
$$

where

$$
\mathcal{W}_{2,0}=\sum_{j<k} \frac{\kappa}{2}\left(\kappa m_{j} m_{k}-1\right)\left(m_{j}+m_{k}\right) \frac{\alpha\left(Z_{j}\right)+\alpha\left(Z_{k}\right)}{\left(Z_{j}-Z_{k}\right)^{2}}
$$


and

$$
\begin{aligned}
\mathcal{W}_{2,1}=\sum_{j<k} \kappa\left(\frac{1}{2}\left(\kappa m_{j} m_{k}-1\right)\left(m_{k}-m_{j}\right) \frac{\alpha\left(Z_{j}\right)}{\left(Z_{j}-Z_{k}\right)^{2}}\right. & \\
& \left.+\frac{m_{k} \beta_{m_{j}}\left(Z_{j}\right)-m_{j} \beta_{m_{k}}\left(Z_{k}\right)}{Z_{j}-Z_{k}}\right) .
\end{aligned}
$$

Recalling (13) we find that the symmetric part gives us the two-body terms we want, up to a constant,

$$
\mathcal{W}_{2,0}=\sum_{j<k} \frac{\kappa}{2}\left(\kappa m_{j} m_{k}-1\right)\left(m_{j}+m_{k}\right)\left(W\left(x_{j}, x_{k}\right)+\alpha_{2}\right) .
$$

Inserting (18) and (9) a straightforward but somewhat tedious computation shows that the terms in the antisymmetric term add up to a constant,

$$
\mathcal{W}_{2,1}=\sum_{j<k} \kappa\left(\frac{\alpha_{2}}{2}\left(\kappa m_{j} m_{k}+1\right)\left(m_{j}+m_{k}\right)+\left(\beta_{1}-\alpha_{2}(1+\kappa)\right) m_{j} m_{k}\right) .
$$

Inserting the second equation in (19) and (18) a simple computation shows that the one-body terms are identical with

$$
\mathcal{W}_{1}=\sum_{j} \frac{1}{m_{j}} V_{m_{j}}\left(X_{j}\right)
$$

with $V_{m}$ defined in (17). Collecting all terms we obtain the identity in (23) with the constant

$$
\begin{aligned}
\mathcal{E}_{0}=-2 \kappa^{2} \alpha_{2} \sum_{j<k<\ell} m_{j} m_{k} m_{\ell}-\kappa^{2} \alpha_{2} \sum_{j<k} m_{j} m_{k}\left(m_{j}+m_{k}\right) & \\
& -\kappa\left(\beta_{1}-\alpha_{2}(1+\kappa)\right) \sum_{j<k} m_{j} m_{k} .
\end{aligned}
$$

Using the notation in (22) we find by straightforward computations that this constant is identical with the one given in (21).

The physical interpretation of $\mathcal{H}$ in (16) as Schrödinger operator of a quantum many-body system requires that it defines a self-adjoint Hilbert space operator bounded from below. This is the case under certain obvious restrictions on parameters. We now discuss this Hilbert space structure for the different cases listed in Table 1, but our discussion will be brief since this aspect does not play any role for our construction in the following sections.

In cases I, III and VII the relevant Hilbert space is $L^{2}\left(\mathbb{R}^{\mathcal{N}}\right)$, in case IV we have instead $L^{2}\left(\mathbb{R}_{+}^{\mathcal{N}}\right)$, while $L^{2}\left([-\pi, \pi]^{\mathcal{N}}\right)$ is associated to case II, and $L^{2}\left([0, \pi]^{\mathcal{N}}\right)$ to cases $\mathrm{V}$ and VI (the weight function is in all these cases constant and equal to 1). It is obvious in all these cases that $Q_{j}^{+}$in the proof above is the Hilbert space adjoint of $Q_{j}^{-}$(on suitable domains), and thus, if all $m_{j}$ are positive, that $\mathcal{H}$ in (23) defines a unique self-adjoint operator via the Friedrichs extension (see, e.g., [43]) with $\Phi_{0}$ as groundstate provided that $\Phi_{0}$ is square integrable. In particular, the $H_{N}$ in (3) define, under obvious restrictions on parameters, self-adjoint operators bounded from below. However, this is not the case for the deformed operators (6). 
2.2. Important special cases. We proceed to discuss the special cases of Proposition 2.1 which underlies our construction of eigenfunctions of the differential operators (3) and (6) in Sections 3 and 4 respectively. Setting $\mathcal{N}=N, m_{j}=1$ and $X_{j}=x_{j}$ for $j=1, \ldots, N$ we obtain as a first special case the following:

Corollary 2.1. The function $\Psi_{0}$, as defined by (5) and (12), is an eigenfunction of the Schrödinger operator (3) with corresponding eigenvalue $E_{0}$.

The remarkable identity underlying our solution method in Section 3 is obtained from Proposition 2.1 by choosing $\mathcal{N}=2 N, m_{j}=1, X_{j}=x_{j}, m_{N+j}=-1$, and $X_{N+j}=y_{j}$ for $j=1, \ldots, N$. We observe that $\mathcal{H}$ in (16) then becomes a difference of two Schrödinger operators (3). Denoting $\Phi_{0}$ as $F$ and $\mathcal{E}_{0}$ as $C_{N}$ we obtain the following:

Corollary 2.2. Let $H_{N}(\mathbf{x})=H_{N}$ be the operator in (3),

$$
H_{N}^{(-)}(\mathbf{y})=\sum_{j=1}^{N}\left(-\partial_{y_{j}}^{2}+V_{-1}\left(y_{j}\right)\right)+\kappa(\kappa-1) \sum_{j<k} W\left(y_{j}, y_{k}\right)
$$

and

$$
F(\mathbf{x}, \mathbf{y})=\prod_{j=1}^{N} \psi_{0}\left(x_{j}\right) \psi_{0,-1}\left(y_{j}\right) \frac{\prod_{j<k}\left(z_{k}-z_{j}\right)^{\kappa}\left(w_{k}-w_{j}\right)^{\kappa}}{\prod_{j, k}\left(w_{k}-z_{j}\right)^{\kappa}}
$$

with $z_{j}=z\left(x_{j}\right), w_{j}=z\left(y_{j}\right)$, and $V_{-1}(x)$ and $\psi_{0,-1}(x)$ as in (17) and (19) for $m=-1$. Then

$$
\left(H_{N}(\mathbf{x})-H_{N}^{(-)}(\mathbf{y})-C_{N}\right) F(\mathbf{x}, \mathbf{y})=0
$$

with the constant

$$
C_{N}=\kappa\left(\beta_{1}-\alpha_{2}(1+\kappa)\right) N .
$$

It is important to note that also $H_{N}^{(-)}$is a Schrödinger operator of the same kind as $H_{N}$, only the coupling parameters are different. For the convenience of the reader we give the modified parameters for our examples I and III-VII,

$$
\omega_{-1}=-\omega, \quad c_{-1}=-\kappa-c, \quad a_{-1}=\kappa-a, \quad b_{-1}=\kappa-b .
$$

The key property that makes the special case of Proposition 2.1] stated in Corollary 2.2 interesting as a tool for constructing the eigenfunctions of a Schrödinger operator (3) is that the variables $\mathbf{X}$ of $\mathcal{H}$ in (16) are divided in two groups $\mathbf{x}$ and $\mathbf{y}$ such that the interaction terms involving variables belonging to different groups all vanish. It is interesting to note that there are other cases where this happens. For example, the number of particles with parameters $m_{j}=1$ and $m_{j}=-1$ need not be the same. More generally, if we divide the variables $\mathbf{X}$ into two groups where the 'mass parameters' in the first group are $m_{j}=1$ or $-1 / \kappa$ and in the second group $m_{k}=-1$ or $1 / \kappa$, then all interaction terms between particles in different groups vanish (since $\frac{1}{2} \kappa\left(m_{j}+m_{k}\right)\left(\kappa m_{j} m_{k}-1\right)=0$ in all these cases). We then obtain an identity as in (25) but now involving two deformed Calogero-Sutherland operators (6) which can have, in general, different particle numbers. Such an identity involves four different kinds of particles, and the number of particles of each kind can be arbitrary. These identities will allow us to construct eigenfunctions of the deformed Calogero-Sutherland operators (6) in Section 4 . The most general such identity corresponds to the following special case of Proposition 2.1. 
$\mathcal{N}=N+\tilde{N}+M+\tilde{M}$ with non-negative integers $N, \tilde{N}, M, \tilde{M}$ such that $\mathcal{N}$ is non-zero, $m_{j}=1$ and $X_{j}=x_{j}$ for $j=1,2, \ldots, N, m_{N+J}=-1 / \kappa$ and $X_{N+J}=\tilde{x}_{J}$ for $J=1,2, \ldots, \tilde{N}, m_{N+\tilde{N}+k}=-1$ and $X_{N+\tilde{N}+k}=y_{k}$ for $k=1,2, \ldots, M$, and $m_{N+\tilde{N}+M+K}=1 / \kappa$ and $X_{N+\tilde{N}+M+K}=\tilde{y}_{K}$ for $K=1,2, \ldots, \tilde{M}$.

Corollary 2.3. Let $H_{N, \tilde{N}}(\mathbf{x}, \tilde{\mathbf{x}})=H_{N, \tilde{N}}$ be the operator in (6) with $\tilde{V}=V_{-1 / \kappa}$,

$$
\begin{aligned}
H_{M, \tilde{M}}^{(-)}(\mathbf{y}, \tilde{\mathbf{y}})= & \sum_{k=1}^{M}\left(-\partial_{y_{k}}^{2}+V_{-1}\left(y_{k}\right)\right)-\sum_{K=1}^{\tilde{M}} \kappa\left(-\partial_{\tilde{y}_{K}}^{2}+V_{1 / \kappa}\left(\tilde{y}_{K}\right)\right) \\
& +\kappa(\kappa-1) \sum_{j<k} W\left(y_{j}, y_{k}\right)+\frac{\kappa-1}{\kappa} \sum_{J<K} W\left(\tilde{y}_{j}, \tilde{y}_{k}\right) \\
& +(1-\kappa) \sum_{j, K} W\left(y_{j}, \tilde{y}_{K}\right)
\end{aligned}
$$

where $M$ and $\tilde{M}$ are arbitrary non-negative integers, and

$$
\begin{aligned}
F_{N, \tilde{N}, M, \tilde{M}}(\mathbf{x}, \tilde{\mathbf{x}}, \mathbf{y}, \tilde{\mathbf{y}}) & =\prod_{j=1}^{N} \psi_{0}\left(x_{j}\right) \prod_{J=1}^{\tilde{N}} \psi_{0,-1 / \kappa}\left(\tilde{x}_{J}\right) \prod_{k=1}^{M} \psi_{0,-1}\left(y_{k}\right) \prod_{K=1}^{\tilde{M}} \psi_{0,1 / \kappa}\left(\tilde{y}_{K}\right) \\
& \times \frac{\prod_{j<k}\left(z_{k}-z_{j}\right)^{\kappa} \prod_{J<K}\left(\tilde{z}_{K}-\tilde{z}_{J}\right)^{1 / \kappa}}{\prod_{j, J}\left(\tilde{z}_{J}-z_{j}\right)} \\
& \times \frac{\prod_{j<k}\left(w_{k}-w_{j}\right)^{\kappa} \prod_{J<K}\left(\tilde{w}_{K}-\tilde{w}_{J}\right)^{1 / \kappa}}{\prod_{j, J}\left(\tilde{w}_{J}-w_{j}\right)} \\
& \times \frac{\prod_{j, K}\left(\tilde{w}_{K}-z_{j}\right) \prod_{J, k}\left(w_{k}-\tilde{z}_{J}\right)}{\prod_{j, k}\left(w_{k}-z_{j}\right)^{\kappa} \prod_{J, K}\left(\tilde{w}_{K}-\tilde{z}_{J}\right)^{1 / \kappa}}
\end{aligned}
$$

with $z_{j}=z\left(x_{j}\right), w_{j}=z\left(y_{j}\right), \tilde{z}_{J}=z\left(\tilde{x}_{J}\right), \tilde{w}_{J}=z\left(\tilde{y}_{J}\right)$, and $V_{m}(x)$ and $\psi_{0, m}(x)$ as in (17) and (19), respectively, for $m=-1$ and $m= \pm 1 / \kappa$. Then

$$
\left(H_{N, \tilde{N}}(\mathbf{x}, \tilde{\mathbf{x}})-H_{M, \tilde{M}}^{(-)}(\mathbf{y}, \tilde{\mathbf{y}})-C_{N, \tilde{N}, M, \tilde{M}}\right) F_{N, \tilde{N}, M, \tilde{M}}(\mathbf{x}, \tilde{\mathbf{x}}, \mathbf{y}, \tilde{\mathbf{y}})=0
$$

with the constant

$$
\begin{aligned}
C_{N, \tilde{N}, M, \tilde{M}}=-\frac{\kappa^{2} \alpha_{2}}{3} & \left(\left(N_{-}-\tilde{N}_{-} / \kappa\right)^{3}-N_{-}+\tilde{N}_{-} / \kappa^{3}\right) \\
& -\frac{\kappa\left(\beta_{1}-(1+\kappa) \alpha_{2}\right)}{2}\left(\left(N_{-}-\tilde{N}_{-} / \kappa\right)^{2}-N_{+}-\tilde{N}_{+} / \kappa^{2}\right),
\end{aligned}
$$

where

$$
N_{ \pm}=N \pm M, \quad \tilde{N}_{ \pm}=\tilde{N} \pm \tilde{M} .
$$

Using this identity we can straightforwardly generalise our solution method and obtain many different formulae for eigenfunctions of the deformed CalogeroSutherland operators ([6): for fixed particle numbers $N$ and $\tilde{N}$ one is free to choose $M$ and $\tilde{M}$ arbitrarily and each choice gives a family of eigenfunctions labelled by integer vectors $\mathbf{n} \in \mathbb{Z}^{M+\tilde{M}}$. As discussed in Section 4.2, we obtain in this way a number of series representations for each eigenfunction, a fact which is interesting already in the standard (non-deformed) case $\tilde{N}=0$. 
2.3. Case II. In order to put the general discussion in the previous two sections into perspective we consider in some detail the special case II; see Tables 11 and 2 The Schrödinger operator (3) is in this case given by

$$
H_{N}=-\sum_{j=1}^{N} \partial_{x_{j}}^{2}+2 \kappa(\kappa-1) \sum_{j<k} \frac{1}{4 \sin ^{2}\left(\frac{x_{j}-x_{k}}{2}\right)} .
$$

As we mentioned in the introduction, this Schrödinger operator was introduced and studied by Sutherland [50,51], and its eigenfunctions are given by the so-called Jack polynomials. In this special case the identity in Corollary 2.2 is equivalent to a well-known and important identity in the theory of Jack polynomials, and Corollary 2.3 gives a natural generalisation of this identity to the deformed case. More precisely, we have the following:

Corollary 2.4. Let

$$
F(\mathbf{x}, \mathbf{y})=\prod_{j=1}^{N} w_{j}^{\kappa} \frac{\prod_{j<k}\left(z_{k}-z_{j}\right)^{\kappa}\left(w_{k}-w_{j}\right)^{\kappa}}{\prod_{j, k}\left(w_{k}-z_{j}\right)^{\kappa}}
$$

with $z_{j}=e^{i x_{j}}$ and $w_{j}=e^{i y_{j}}$. Then

$$
\left(H_{N}(\mathbf{x})-H_{N}(\mathbf{y})\right) F(\mathbf{x}, \mathbf{y})=0,
$$

where the arguments indicate that the Schrödinger operator acts in the variables $\mathbf{x}$ and $\mathbf{y}$, respectively.

As mentioned above, the identity (26), and in particular the function $F$, is directly related with the theory of Jack polynomials. In order to make this precise we observe that

$$
F(\mathbf{z}, \mathbf{w})=\prod_{j<k}\left(z_{k}-z_{j}\right)^{\kappa}\left(1 / w_{j}-1 / w_{k}\right)^{\kappa} \Pi(\mathbf{z}, \mathbf{w})
$$

with the function $\Pi$ given by

$$
\Pi(\mathbf{z}, \mathbf{w})=\frac{1}{\prod_{j, k}\left(1-z_{j} / w_{k}\right)^{\kappa}} .
$$

It is well known that this latter function $\Pi$ has the following expansion in the monic Jack polynomials $\mathcal{J}_{\lambda}$ :

$$
\Pi(\mathbf{z}, \mathbf{w})=\sum_{\boldsymbol{\lambda}} b_{\boldsymbol{\lambda}} \mathcal{J}_{\boldsymbol{\lambda}}(\mathbf{z}) \mathcal{J}_{\boldsymbol{\lambda}}\left(\mathbf{w}^{-1}\right)
$$

for some (explicitly known) coefficients $b_{\boldsymbol{\lambda}}$, and where $\mathbf{w}^{-1}=\left(1 / w_{1}, \ldots, 1 / w_{N}\right)$; see, e.g., Sections VI.4 and VI.10 in [39]. We recall that the monic Jack polynomials are usually denoted $P_{\boldsymbol{\lambda}}$ rather than $\mathcal{J}_{\boldsymbol{\lambda}}$. We have used the latter notation in order to avoid clashes with notation used in later sections. Up to degeneracies in the spectrum of the Schrödinger operator $H_{N}$, this expansion can be deduced as a consequence of the identity (26). To establish this fact we let $\Psi_{0}(\mathbf{z})=\prod_{j<k}\left(z_{k}-\right.$ $\left.z_{j}\right)^{\kappa}$, and similarly for $\Psi_{0}\left(\mathbf{w}^{-1}\right)$. It is well known that the functions $\Psi_{0}(\mathbf{z}) \mathcal{J}_{\boldsymbol{\lambda}}(\mathbf{z})$ are eigenfunctions of $H_{N}$; this can for example be inferred from results of Stanley [49]. Since $H_{N}(\mathbf{y})$ is invariant under the substitution of $-y_{j}$ for $y_{j}$ which maps $w_{j}$ to $1 / w_{j}$, the functions $\Psi_{0}\left(\mathbf{w}^{-1}\right) \mathcal{J}_{\boldsymbol{\lambda}}\left(\mathbf{w}^{-1}\right)$ are eigenfunctions of the same Schrödinger operator $H_{N}$, and with the same eigenvalues. We observe that $\Pi(\mathbf{z}, \mathbf{w})$ is invariant 
under permutations of both the variables $\mathbf{z}$ as well as the variables $\mathbf{w}$. It follows that it has an expansion in Jack polynomials of the form

$$
\Pi(\mathbf{z}, \mathbf{w})=\sum_{\boldsymbol{\lambda}} Q_{\boldsymbol{\lambda}}(\mathbf{z}) \mathcal{J}_{\boldsymbol{\lambda}}\left(\mathbf{w}^{-1}\right)
$$

where the functions $Q_{\boldsymbol{\lambda}}$ are to be determined. Since $\Psi_{0}\left(\mathbf{w}^{-1}\right) \mathcal{J}_{\boldsymbol{\lambda}}\left(\mathbf{w}^{-1}\right)$ is an eigenfunction of $H_{N}$, the identity (26) implies that also $\Psi_{0}(\mathbf{z}) Q_{\boldsymbol{\lambda}}(\mathbf{z})$ is an eigenfunction of $H_{N}$, and with the same eigenvalue. It follows that, unless this eigenvalue is degenerate, $Q_{\boldsymbol{\lambda}}(\mathbf{z})$ is proportional to $\mathcal{J}_{\boldsymbol{\lambda}}(\mathbf{z})$. We mention that although such degeneracies do occur they are quite rare in this case.

The identities obtained in the previous section thus suggest that there exist expansions, similar to the one given above, for each function $F$ corresponding to a Schrödinger operator (3) or a deformed counterpart (6). However, in most cases the Schrödinger operator $H_{N}$, as well as its deformed counterpart $H_{N, \tilde{N}}$, is not invariant under a change of coordinates mapping $w_{j}$ to $1 / w_{j}$. This explains the appearance of the operator $H_{N}^{(-)}$and the constant $C_{N}$ in Corollary 2.2, as well as that of $H_{M, \tilde{M}}^{(-)}$and $C_{N, \tilde{N}, M, \tilde{M}}$ in Corollary 2.3 .

\section{Eigenfunctions of Calogero-Sutherland type models}

In this section we construct our series representations of the eigenfunctions of the Schrödinger operators (3). These series representations are in terms of the functions $f_{\mathbf{n}}$ defined in (7). As we will see, the identities deduced in the previous section play a central role in this construction. These series representation generalises those obtained in [30] for the special case II and in [20] for I and IV (c.f. Table 1).

3.1. Construction of eigenfunctions. We fix the polynomials $\alpha, \beta$ in (9) and the coupling constant $\kappa>0$ and consider the resulting Schrödinger operator $H_{N}$ as defined by (3), (10), (111) and (13). We observe that the eigenfunctions in (4) are completely determined by the functions $P_{\mathbf{n}}$ in $N$ variables $\mathbf{z}$, and we will refer to the latter as reduced eigenfunctions of $H_{N}$. We also observe that $P_{\mathbf{n}}$ is a reduced eigenfunction of $H_{N}$ if and only if it is an eigenfunction of the differential operator

$$
\tilde{H}_{N}:=\Psi_{0}^{-1}\left(H_{N}-E_{0}\right) \Psi_{0}=-\sum_{j=1}^{N}\left(\alpha\left(z_{j}\right) \partial_{z_{j}}^{2}+\beta\left(z_{j}\right) \partial_{z_{j}}\right)-2 \kappa \sum_{j \neq k} \frac{\alpha\left(z_{j}\right)}{z_{j}-z_{k}} \partial_{z_{j}},
$$

where the last equality follows by a straightforward computation using the fact that $\Psi_{0}$ is an eigenstate of $H_{N}$ with eigenvalue $E_{0}$ and the identities in (8), (13), and (11). Using the identity in Corollary 2.2 it is now straightforward to compute the action of this differential operator on the polynomials $f_{\mathbf{n}}$. In order to state this action in a simple form, and also to facilitate a discussion of its implications, we first introduce some convenient notation. An important ingredient in our construction is the following partial ordering of integer vectors $\mathbf{n}, \mathbf{m} \in \mathbb{Z}^{N}$ :

$$
\mathbf{m} \preceq \mathbf{n} \Leftrightarrow m_{j}+\cdots+m_{N} \leq n_{j}+\cdots+n_{N}, \quad \forall j=1,2, \ldots, N .
$$

That this is only a partial ordering is easily seen, e.g., (522) and (441) are incomparable. The algebra of symmetric polynomials $P(\mathbf{z})$ in $N$ variables $\mathbf{z}=\left(z_{1}, \ldots, z_{N}\right)$ with complex coefficients is denoted $\Lambda_{N}$. The linear subspace consisting of the homogeneous symmetric polynomials of degree $n$, together with the zero polynomial, 
is denoted by $\Lambda_{N}^{n}$. We also denote as $\mathbf{e}_{j}$ the standard basis in $\mathbb{Z}^{N}$, i.e., $\left(\mathbf{e}_{j}\right)_{k}=\delta_{j k}$ for all $j, k=1,2, \ldots, N$, and let

$$
\mathbf{E}_{j, k}^{p, \nu}=(1-\nu) \mathbf{e}_{j}+(1-p+\nu) \mathbf{e}_{k} .
$$

As before,

$$
|\mathbf{n}|:=n_{1}+\ldots+n_{N} .
$$

For simplicity of notation we shall to each $\mathbf{n} \in \mathbb{Z}^{N}$ associate the shifted integer vector $\mathbf{n}^{+}=\left(n_{1}^{+}, \ldots, n_{N}^{+}\right)$with

$$
n_{j}^{+}=n_{j}+\kappa(N+1-j) .
$$

We are now ready to state and prove the following:

Lemma 3.1. For each $\mathbf{n} \in \mathbb{Z}^{N}$,

$$
\begin{aligned}
& \tilde{H}_{N} f_{\mathbf{n}}=\left(E_{\mathbf{n}}-E_{0}\right) f_{\mathbf{n}} \\
& -\sum_{j=1}^{N}\left(\alpha_{1} n_{j}^{+}\left(n_{j}^{+}-1\right)+\left(\beta_{0}-(1+\kappa) \alpha_{1}\right)\left(n_{j}^{+}-1\right)\right) f_{\mathbf{n}-\mathbf{e}_{j}} \\
& \quad-\alpha_{0} \sum_{j=1}^{N}\left(n_{j}^{+}-1\right)\left(n_{j}^{+}-2\right) f_{\mathbf{n}-2 \mathbf{e}_{j}} \\
& \quad+\kappa(\kappa-1) \sum_{j<k} \sum_{p=0}^{2} \sum_{\nu=1}^{\infty} \alpha_{p}(2 \nu-p) f_{\mathbf{n}-\mathbf{E}_{j, k}^{p, \nu} .}
\end{aligned}
$$

Proof. Note that the function $F$ in Corollary 2.2 can be written as follows:

$$
F(\mathbf{x}, \mathbf{y})=\Psi_{0}(\mathbf{x}) G(\mathbf{y}) \prod_{j=1}^{N} w_{j}^{-\kappa(N+1-j)} \frac{\prod_{j<k}\left(1-w_{j} / w_{k}\right)^{\kappa}}{\prod_{j, k}\left(1-z_{j} / w_{k}\right)^{\kappa}}
$$

with

$$
G(\mathbf{y}):=\prod_{j=1}^{N} \psi_{0,-1}\left(y_{j}\right) .
$$

The definition (7) of the polynomials $f_{\mathbf{n}}$ thus implies

$$
F(\mathbf{x}, \mathbf{y})=\Psi_{0}(\mathbf{x}) G(\mathbf{y}) \sum_{\mathbf{n} \in \mathbb{Z}^{N}} f_{\mathbf{n}}(\mathbf{z}) \mathbf{w}^{-\mathbf{n}^{+}} .
$$

It follows from Corollary 2.2 that

$$
\sum_{\mathbf{n} \in \mathbb{Z}^{N}}\left(\tilde{H}_{N} f_{\mathbf{n}}(\mathbf{z})\right) \mathbf{w}^{-\mathbf{n}^{+}}=\sum_{\mathbf{n} \in \mathbb{Z}^{N}} f_{\mathbf{n}}(\mathbf{z})\left(\bar{H}_{N}^{(-)}+C_{N}\right) \mathbf{w}^{-\mathbf{n}^{+}}
$$

where

$$
\bar{H}_{N}^{(-)}:=G^{-1}(\mathbf{y}) H_{N}^{(-)} G(\mathbf{y})
$$

Using (8), (13), and (11), as well as the definition of $H_{N}^{(-)}$, we find that

$$
\bar{H}_{N}^{(-)}=-\sum_{j=1}^{N}\left(\alpha\left(w_{j}\right) \partial_{w_{j}}^{2}+\beta_{-1}\left(w_{j}\right) \partial_{w_{j}}\right)+\kappa(\kappa-1) \sum_{j<k}\left(\frac{\alpha\left(w_{j}\right)+\alpha\left(w_{k}\right)}{\left(w_{j}-w_{k}\right)^{2}}-\alpha_{2}\right)
$$

with

$$
\beta_{-1}(w)=-\beta(w)+(1+\kappa) \alpha^{\prime}(w)
$$


Recalling (9) we expand the interaction terms in Laurent series and obtain

$$
\begin{aligned}
\frac{\alpha\left(w_{j}\right)+\alpha\left(w_{k}\right)}{\left(w_{j}-w_{k}\right)^{2}}-\alpha_{2} & =\frac{2 \alpha_{2} w_{j} w_{k}+\alpha_{1}\left(w_{j}+w_{k}\right)+2 \alpha_{0}}{w_{k}^{2}\left(1-w_{j} / w_{k}\right)^{2}} \\
& =\sum_{p=0}^{2} \sum_{\nu=1}^{\infty} \alpha_{p}(2 \nu-p) \frac{w_{j}^{\nu-1}}{w_{k}^{\nu+1-p}}
\end{aligned}
$$

valid for $\left|w_{N}\right|>\left|w_{N-1}\right|>\cdots>\left|w_{1}\right|>\max _{k}\left(\left|z_{k}\right|\right)$. It is now straightforward to compute the r.h.s. of (33), and by comparing the coefficients of $\mathbf{w}^{-\mathbf{n}^{+}}$on both sides of the resulting equation we obtain (32) with

$$
E_{\mathbf{n}}=-\sum_{j=1}^{N}\left[\alpha_{2} n_{j}^{+}\left(n_{j}^{+}+1\right)+\left(\beta_{1}-2(1+\kappa) \alpha_{2}\right) n_{j}^{+}\right]+C_{N} .
$$

By straightforward computations we find that the latter coincides with $E_{\mathbf{n}}$ in (14) and (15).

It is clear that the action of $\tilde{H}_{N}$ on the polynomials $f_{\mathbf{n}}$ is triangular in the sense that $\tilde{H}_{N} f_{\mathbf{n}}$ is a linear combination of polynomials $f_{\mathbf{m}}$ with $\mathbf{m} \preceq \mathbf{n}$. In fact, depending on the specific choice of $\alpha$ and $\beta$, the set of possible $\mathbf{m}$ is in many cases significantly smaller. In any case, this triangular structure suggests that there exist eigenfunctions, with corresponding eigenvalues $E_{\mathbf{n}}-E_{0}$, of the differential operator $\tilde{H}_{N}$ which are of the form

$$
P_{\mathbf{n}}=f_{\mathbf{n}}+\sum_{\mathbf{m}} u_{\mathbf{n}}(\mathbf{m}) f_{\mathbf{m}}
$$

where the sum is over integer vectors $\mathbf{m} \prec \mathbf{n}$. Indeed, if we take this as an ansatz then we find that this is the case if and only if the coefficients satisfy the recursion relation

$$
\begin{aligned}
& \left(E_{\mathbf{n}}-E_{\mathbf{m}}\right) u_{\mathbf{n}}(\mathbf{m})=-\sum_{j=1}^{N} m_{j}^{+}\left(\beta_{0}+\alpha_{1}\left(m_{j}^{+}-\kappa\right)\right) u_{\mathbf{n}}\left(\mathbf{m}+\mathbf{e}_{j}\right) \\
& -\alpha_{0} \sum_{j=1}^{N} m_{j}^{+}\left(m_{j}^{+}+1\right) u_{\mathbf{n}}\left(\mathbf{m}+2 \mathbf{e}_{j}\right)+\kappa(\kappa-1) \sum_{j<k} \sum_{p=0}^{2} \alpha_{p}(2 \nu-p) u_{\mathbf{n}}\left(\mathbf{m}+\mathbf{E}_{j, k}^{p, \nu}\right) .
\end{aligned}
$$

Since $u_{\mathbf{n}}(\mathbf{n})$ is fixed to one, it is clear that if $E_{\mathbf{n}}-E_{\mathbf{m}} \neq 0$ for all $\mathbf{m}$ which appear in (34), then this recursion relation uniquely determines the remaining coefficients $u_{\mathbf{n}}(\mathbf{m})$. This condition of non-degeneracy on the eigenvalues is generically satisfied, but there exist special cases and specific integer vectors $\mathbf{n}$ for which it fails to hold true; see Remark 3.2 for a further discussion of this point.

3.2. Case II. In order to draw attention to some of the specific features of the action of $\tilde{H}_{N}$ on the polynomials $f_{\mathbf{n}}$ we consider in some detail the special case II; see Tables 1 and 2. On the one hand this is in many ways the simplest non trivial case, and our construction of reduced eigenfunctions is therefore particularly simple in this case, but, on the other hand, it already contains many of the key features of the general case. For the special case II we have $\alpha(z)=-z^{2}$ and $\beta(z)=-z$, and 
consequently that

$$
\tilde{H}_{N} f_{\mathbf{n}}=\left(E_{\mathbf{n}}-E_{0}\right) f_{\mathbf{n}}-2 \kappa(\kappa-1) \sum_{j<k} \sum_{\nu=1}^{\infty} \nu f_{\mathbf{n}-\nu\left(\mathbf{e}_{k}-\mathbf{e}_{j}\right)} .
$$

Clearly, the right hand side now only contains polynomials $f_{\mathbf{m}}$ such that $|\mathbf{m}|=|\mathbf{n}|$. It is interesting to compare this expression with the corresponding action of $\tilde{H}_{N}$ on the monomial symmetric polynomials

$$
m_{\boldsymbol{\lambda}}(\mathbf{z}):=\sum_{P} z_{1}^{\lambda_{P(1)}} \cdots z_{N}^{\lambda_{P(N)}}
$$

with $\boldsymbol{\lambda}$ a partition of length $\ell(\boldsymbol{\lambda}) \leq N$, and where the sum extends over all distinct permutations $P$ of the parts $\lambda_{j}$ of $\boldsymbol{\lambda}$. To this end we let $\lfloor x\rfloor$ denote the integer part of $x \in \mathbb{R}$. In addition, for any $\mathbf{n} \in \mathbb{N}_{0}^{N}$ we identify $m_{\mathbf{n}}$ with the unique $m_{\boldsymbol{\lambda}}$ such that $\mathbf{n}=P(\boldsymbol{\lambda})$ for some permutation $P$. Using the identity

$$
\frac{1}{x-y}\left(x^{2} \partial_{x}-y^{2} \partial_{y}\right)\left(x^{n} y^{m}+x^{m} y^{n}\right)=(n-m) \sum_{k=0}^{n-m} x^{n-k} y^{m+k}+2 m x^{n} y^{m},
$$

valid for any non-negative integers $n$ and $m$ such that $n \geq m$, it is now easy to verify that

$$
\tilde{H}_{N} m_{\boldsymbol{\lambda}}=\left(E_{\boldsymbol{\lambda}}-E_{0}\right) m_{\boldsymbol{\lambda}}+2 \kappa \sum_{j<k}\left(\lambda_{j}-\lambda_{k}\right) \sum_{\nu=0}^{\left\lfloor\left(\lambda_{j}-\lambda_{k}\right) / 2\right\rfloor} m_{\boldsymbol{\lambda}+\nu\left(\mathbf{e}_{k}-\mathbf{e}_{j}\right) .}
$$

We note, in particular, that the action of $\tilde{H}_{N}$ on the polynomials $f_{\mathbf{n}}$ does not contain an explicit dependence on the integer vector $\mathbf{n}$. In contrast, the action on the symmetric monomials $m_{\boldsymbol{\lambda}}$ do contain an explicit dependence on the partition $\boldsymbol{\lambda}$. It is also interesting to note that the corresponding recursion relation (35) can in fact be solved to yield the following explicit series representation for the eigenfunctions of $\tilde{H}_{N}$ :

$$
\begin{aligned}
& P_{\mathbf{n}}=f_{\mathbf{n}}+\sum_{s=1}^{\infty}(2 \kappa(1-\kappa))^{s} \sum_{j_{1}<k_{1}} \sum_{\nu_{1}=1}^{\infty} \nu_{1} \times \cdots \times \sum_{j_{s}<k_{s}} \sum_{\nu_{s}=1}^{\infty} \nu_{s}
\end{aligned}
$$

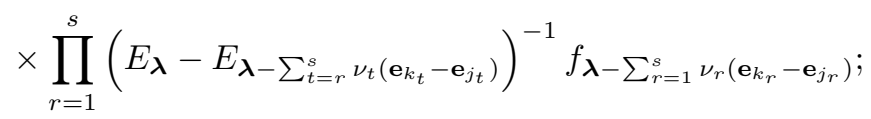

see [31] for further details. It is important to note that the fact that $f_{\mathbf{n}}$ is non-zero only if $\mathbf{n} \succeq 0$ (see Corollary 3.1) implies that this series only contains a finite number of terms, and thus is well-defined. Even though it can be done in principle, it seems harder to solve the recursion relations resulting from the use of the monomial symmetric polynomials. Detailed discussions of the above construction of series representations for the reduced eigenfunctions of the Schrödinger operator (3) in the cases I and IV can be found in $[17,20]$. We also mention that it is possible to write down explicit formulae for the reduced eigenfunctions of the Schrödinger operator (3) in the general case which, however, are somewhat involved] 1

\footnotetext{
${ }^{1}$ The interested reader can find these formulae in the first arXiv version of the present paper; see http://arxiv.org/abs/math-ph/0703090v1
} 
3.3. Completeness of the reduced eigenfunctions. In Section 3.1 we constructed, under a certain condition of non-degeneracy on the corresponding eigenvalues, for each integer vector $\mathbf{n} \in \mathbb{Z}^{N}$ a reduced eigenfuntion $P_{\mathbf{n}}$ of the Schrödinger operator $H_{N}$. In this section we show that if we restrict attention to integer vectors $\mathbf{n}=\boldsymbol{\lambda}$ for some partition $\boldsymbol{\lambda}$, then the corresponding reduced eigenfunctions form a linear basis for $\Lambda_{N}$. The idea of the proof is to first establish that the expansion of the symmetric polynomials $f_{\mathbf{n}}$ in terms of a particular set of symmetric polynomials $g_{\boldsymbol{\lambda}}$, known to constitute such a basis, has a particular triangular structure. This implies that the $f_{\boldsymbol{\lambda}}$, labelled by the partitions $\boldsymbol{\lambda}$ of length $\ell(\boldsymbol{\lambda}) \leq N$, form a linear basis for $\Lambda_{N}$. We then obtain a similar result for the reduced eigenfunctions $P_{\boldsymbol{\lambda}}$ by showing that they are, in the same sense, triangular in the $f_{\boldsymbol{\lambda}}$.

We proceed to recall that the polynomials $g_{\boldsymbol{\lambda}}$ in question, sometimes referred to as the modified complete symmetric polynomials, can be defined by the expansion $\left(\mathbf{w}^{-1}=\left(w_{1}^{-1}, \ldots, w_{N}^{-1}\right)\right)$

$$
\Pi(\mathbf{z}, \mathbf{w})=\sum_{\boldsymbol{\lambda}} g_{\boldsymbol{\lambda}}(\mathbf{z}) m_{\boldsymbol{\lambda}}\left(\mathbf{w}^{-1}\right)
$$

valid for $\min _{k}\left(\left|w_{k}\right|\right)>\max _{j}\left(\left|z_{j}\right|\right)$, and where the sum extends over all partitions $\boldsymbol{\lambda}$ of length $\ell(\boldsymbol{\lambda}) \leq N$. It is well known that the $g_{\boldsymbol{\lambda}}$ constitute a linear basis for $\Lambda_{N}$; see, e.g., Section VI.10 in [39]. Using this fact we now establish the following relation between the $f_{\mathbf{n}}$ and the $g_{\boldsymbol{\lambda}}$, which, in particular, implies that the $f_{\mathbf{n}}$ indeed are well-defined symmetric polynomials:

Lemma 3.2. Let $\mathbf{n} \in \mathbb{Z}^{N}$. Then

$$
f_{\mathbf{n}}=\sum_{\mu} a_{\mathbf{n} \mu} g_{\mu}
$$

for some coefficients $a_{\mathbf{n} \boldsymbol{\mu}}$ and where the sum is over partitions $\boldsymbol{\mu} \preceq \mathbf{n}$ such that $|\boldsymbol{\mu}|=|\mathbf{n}|$. Moreover, if $\mathbf{n}=\boldsymbol{\lambda}$ for some partition $\boldsymbol{\lambda}$ of length $\ell(\boldsymbol{\lambda}) \leq N$, then $a_{\lambda \boldsymbol{\lambda}}=1$.

Proof. It follows from the definitions of the $g_{\boldsymbol{\lambda}}$ and $f_{\mathbf{n}}$ that

$$
\begin{aligned}
\sum_{\mathbf{n} \in \mathbb{Z}^{N}} f_{\mathbf{n}}(\mathbf{z}) \mathbf{w}^{-\mathbf{n}} & =\prod_{j<k}\left(1-w_{j} / w_{k}\right)^{\kappa} \sum_{\mathbf{m} \in \mathbb{N}_{0}^{N}} g_{p(\mathbf{m})}(\mathbf{z}) \mathbf{w}^{-\mathbf{m}} \\
& =\sum_{\mathbf{m} \in \mathbb{N}_{0}^{N}} g_{p(\mathbf{m})}(\mathbf{z}) \prod_{j<k} \sum_{q_{j k}=0}^{\infty}(-1)^{q_{j k}}\left(\begin{array}{c}
\kappa \\
q_{j k}
\end{array}\right) \mathbf{w}^{-\mathbf{m}+\sum_{j<k} q_{j k}\left(\mathbf{e}_{j}-\mathbf{e}_{k}\right)}
\end{aligned}
$$

where $p(\mathbf{m})$ denotes the unique partition obtained by permuting the components of the integer vector $\mathbf{m}$, e.g., $p(2,4,5,2)=(5,4,2,2)$. Comparing the coefficients of $\mathbf{w}^{-\mathbf{n}}$ on both sides of this identity we find that

$$
f_{\mathbf{n}}=\prod_{j<k} \sum_{q_{j k}=0}^{\infty}(-1)^{q_{j k}}\left(\begin{array}{c}
\kappa \\
q_{j k}
\end{array}\right) g_{p\left(\mathbf{n}+\sum_{j<k} q_{j k}\left(\mathbf{e}_{j}-\mathbf{e}_{k}\right)\right)} .
$$

We thus obtained a representation of $f_{\mathbf{n}}$ as a linear superposition of polynomials $g_{\boldsymbol{\mu}}$ where $\boldsymbol{\mu}=p(\mathbf{m})$ for

$$
\mathbf{m}=\mathbf{n}+\sum_{j<k} q_{j k}\left(\mathbf{e}_{j}-\mathbf{e}_{k}\right)
$$

with non-negative integers $q_{j k}$. Obviously, $|\boldsymbol{\mu}|=|\mathbf{m}|=|\mathbf{n}|$ for such $\boldsymbol{\mu}$. To complete the proof we thus only need to show that $\boldsymbol{\mu} \preceq \mathbf{n}$. For that we observe that $\mathbf{m} \preceq \mathbf{n}$. 
Moreover, since $\boldsymbol{\mu}$ is a partition, $\mu_{1} \geq \mu_{2} \geq \cdots \geq \mu_{N}$, and the definition of the ordering (29) thus implies that $\boldsymbol{\mu} \preceq P \boldsymbol{\mu}$ for any permutation $P$ of the parts of $\boldsymbol{\mu}$ and, in particular, $\boldsymbol{\mu} \preceq \mathbf{m}$.

Remark 3.1. It is interesting to note that in the particular case $\kappa=1$ the result in (38) is equivalent to the so-called Jacobi-Trudi identity (originally due to Jacobi [23]), which implies that the well-known Schur polynomials $s_{\boldsymbol{\lambda}}$ are identical with the polynomials $f_{\boldsymbol{\lambda}}$ for $\kappa=1$. The proof of this fact can be obtained as a simple consequence of well-known arguments which can be found in Macdonald's book [39]; see the proof of (3.4") and the preceding discussion in Chapter I.

The fact that each $g_{\boldsymbol{\mu}}$ is a symmetric homogeneous polynomial of degree $|\boldsymbol{\mu}|$ now implies the following:

Corollary 3.1. The function $f_{\mathbf{n}}, \mathbf{n} \in \mathbb{Z}^{N}$, is non-zero only if $\mathbf{n} \succeq \mathbf{0}$. In that case it is a symmetric homogeneous polynomial of degree $|\mathbf{n}|$.

Let $n$ be a non-negative integer and consider the two sets of polynomials $g_{\boldsymbol{\lambda}}$ and $f_{\boldsymbol{\lambda}}$ which are both indexed by those partitions $\boldsymbol{\lambda}$ of $n$ which are of length $\ell(\boldsymbol{\lambda}) \leq N$. Let $K=\left(K_{\boldsymbol{\lambda} \boldsymbol{\mu}}\right)$ denote the transition matrix from the $f_{\boldsymbol{\lambda}}$ to the $g_{\boldsymbol{\lambda}}$, defined by the equalities $f_{\boldsymbol{\lambda}}=\sum_{\boldsymbol{\mu}} K_{\boldsymbol{\lambda} \boldsymbol{\mu}} g_{\boldsymbol{\mu}}$. Given a partition $\boldsymbol{\lambda}$, Lemma 3.2 implies that $K_{\boldsymbol{\lambda} \boldsymbol{\mu}}=0$ unless $\boldsymbol{\mu} \preceq \boldsymbol{\lambda}$ and that $K_{\boldsymbol{\lambda} \boldsymbol{\lambda}}=1$. It is readily verified that the inverse of such a matrix always exist and is of the same form; see, e.g., Section I.6 in [39]. It follows that

$$
g_{\boldsymbol{\lambda}}=f_{\boldsymbol{\lambda}}+\sum_{\mu}\left(K^{-1}\right)_{\boldsymbol{\lambda} \mu} f_{\mu}
$$

where $\left(K^{-1}\right)_{\lambda \mu}$ are the elements in the matrix inverse to $K$ and the sum is over partitions $\boldsymbol{\mu} \prec \boldsymbol{\lambda}$ of $n$. We thus obtain the following:

Proposition 3.1. For any non-negative integer $n$, the polynomials $f_{\boldsymbol{\lambda}}$, labelled by those partitions $\boldsymbol{\lambda}$ of $n$ which are of length $\ell(\boldsymbol{\lambda}) \leq N$, form a linear basis for $\Lambda_{N}^{n}$.

Suppose now that the reduced eigenfunctions $P_{\mathbf{n}}$ of the form (34) exist for all integer vectors $\mathbf{n} \in \mathbb{Z}^{N}$ such that $\mathbf{n}=\boldsymbol{\lambda}$ for some partition $\boldsymbol{\lambda}$. From Lemma 3.2 and (39) we have that any such reduced eigenfunction has a series expansion of the form

$$
P_{\boldsymbol{\lambda}}=f_{\boldsymbol{\lambda}}+\sum_{\mu} v_{\boldsymbol{\lambda} \mu} f_{\mu}
$$

for some coefficients $v_{\boldsymbol{\lambda} \mu}$, and where the sum is over partitions $\boldsymbol{\mu} \prec \boldsymbol{\lambda}$. Fix a partition $\boldsymbol{\lambda}$ of length $\ell(\boldsymbol{\lambda}) \leq N$ and consider the transition matrix from the reduced eigenfunctions $P_{\boldsymbol{\mu}}$ to the polynomials $f_{\boldsymbol{\mu}}$, both indexed by the partitions $\boldsymbol{\mu}$ of length $\ell(\boldsymbol{\mu}) \leq N$ such that $\boldsymbol{\mu} \preceq \boldsymbol{\lambda}$. Observe that its entries are non-zero only if $\boldsymbol{\mu} \preceq \boldsymbol{\lambda}$ and that all diagonal entries are equal to one. It is clear from the arguments leading up to Proposition 3.1 that this transition matrix has a well-defined inverse of the same form. It follows that each polynomial $f_{\boldsymbol{\lambda}}$ is a linear combination of reduced eigenfunctions $P_{\boldsymbol{\mu}}$ with $\boldsymbol{\mu} \preceq \boldsymbol{\lambda}$. Proposition 3.1 thus implies the following:

Proposition 3.2. If the reduced eigenfunctions $P_{\boldsymbol{\lambda}}$ in (34) exist for all partitions $\boldsymbol{\lambda}$ of length $\ell(\boldsymbol{\lambda}) \leq N$ then they form a linear basis for $\Lambda_{N}$.

Remark 3.2. As discussed after Equation (35), a reduced eigenfunction of the form (34) exist if $E_{\mathbf{n}}-E_{\mathbf{m}} \neq 0$ for all $\mathbf{m}$ which appear in (34). It is easy to see that 
this condition of non-degeneracy holds true if $\alpha_{2}=0$ (and $\beta_{1} \neq 0$ ), including the special cases I and IV (see, e.g., [20]). It is also known to hold for all integer vectors $\mathbf{n} \in \mathbb{Z}^{N}$ such that $\mathbf{n}=\boldsymbol{\lambda}$ for some partition $\boldsymbol{\lambda}$ in the special case II (see, e.g., [31]), assuming that $\kappa>0$. It is interesting to note that if $\alpha_{2} \neq 0$ and if one allow $\kappa$ to be negative then there exist parameter values and integer vectors $\mathbf{n}$ such that $E_{\mathbf{n}}=E_{\mathbf{m}}$ for some integer vector $\mathbf{m}$ which appear in (34). We stress, however, that for generic parameter values and integer vectors $\mathbf{n}$ this condition of non-degeneracy on the eigenvalues is satisfied.

\section{Eigenfunctions of Deformed Calogero-Sutherland type models}

In this section we sketch the generalisation of our construction of eigenfunctions from the previous section to the deformed Calogero-Sutherland operators $H_{N, \tilde{N}}$ (6), emphasising the features which are new. Just as the construction in the previous section was based on Corollary 2.2, the discussion below is based on Corollary 2.3. We recall that the identity in this latter corollary relates the operator $H_{N, \tilde{N}}$ to an operator of the same type, but with particle numbers $M$ and $\tilde{M}$. We stress that these particle numbers can be chosen freely, and that, as we will discuss in Section 4.2. the complexity of the resulting series representation of a given eigenfunction can be highly dependent on the specific choice. As described already in the beginning of Section 1 this can be used to construct simpler explicit formulae for many of the eigenfunctions already in the special case $\tilde{N}=0$.

We proceed to describe the structure of the eigenfunctions of the deformed Calogero-Sutherland operators (6). Following Sergeev and Veselov [48] we let $\Lambda_{N, \tilde{N}, \kappa}$ be the algebra of polynomials $P(\mathbf{z}, \tilde{\mathbf{z}})$ in two sets of independent variables $\mathbf{z}=\left(z_{1}, \ldots, z_{N}\right)$ and $\tilde{\mathbf{z}}=\left(\tilde{z}_{1}, \ldots, \tilde{z}_{\tilde{N}}\right)$ with complex coefficients such that they are separately symmetric in the variables $\mathbf{z}$ and $\tilde{\mathbf{z}}$ and, furthermore, obey the condition

$$
\left.\left(\partial_{z_{j}}+\kappa \partial_{\tilde{z}_{J}}\right) P(\mathbf{z}, \tilde{\mathbf{z}})\right|_{z_{j}=\tilde{z}_{J}}=0
$$

for all $j=1, \ldots, N$ and $J=1, \ldots, \tilde{N}$. We will show that the deformed CalogeroSutherland operators (6) have eigenfunctions which are naturally labelled by partitions $\boldsymbol{\lambda}=\left(\lambda_{1}, \lambda_{2}, \ldots\right)$ in the so-called fat $(N, \tilde{N})$-hook, i.e., $\lambda_{N+1} \leq \tilde{N}$, and are of the form

$$
\Psi_{\boldsymbol{\lambda}}(\mathbf{x}, \tilde{\mathbf{x}})=\Psi_{0}(\mathbf{x}, \tilde{\mathbf{x}}) P_{\boldsymbol{\lambda}}(\mathbf{z}, \tilde{\mathbf{z}})
$$

with $z_{j}=z\left(x_{j}\right), \tilde{z}_{J}=z\left(\tilde{x}_{J}\right)$,

$$
\Psi_{0}(\mathbf{x}, \tilde{\mathbf{x}})=\prod_{j=1}^{N} \psi_{0}\left(x_{j}\right) \prod_{J=1}^{\tilde{N}} \psi_{0,-1 / \kappa}\left(\tilde{x}_{J}\right) \frac{\prod_{j<k}\left(z_{k}-z_{j}\right)^{\kappa} \prod_{J<K}\left(\tilde{z}_{K}-\tilde{z}_{J}\right)^{1 / \kappa}}{\prod_{j, J}\left(\tilde{z}_{J}-z_{j}\right)}
$$

and where the $P_{\boldsymbol{\lambda}}$ are particular polynomials contained in the algebra $\Lambda_{N, \tilde{N}, \kappa}$. Moreover, the corresponding eigenvalues are given by

$$
E_{\boldsymbol{\lambda}}=E_{0}-\sum_{j=1}^{\ell(\boldsymbol{\lambda})}\left(\alpha_{2} \lambda_{j}\left(\lambda_{j}-1\right)+\left(2 \alpha_{2}(\kappa(N-j)-\tilde{N})+\beta_{1}\right) \lambda_{j}\right)
$$


with

$$
\begin{aligned}
E_{0}=-\frac{\kappa^{2} \alpha_{2}}{3}\left((N-\tilde{N} / \kappa)^{3}-N+\tilde{N} / \kappa^{3}\right) & \\
& -\frac{\kappa\left(\beta_{1}-(1+\kappa) \alpha_{2}\right)}{2}\left((N-\tilde{N} / \kappa)^{2}-N-\tilde{N} / \kappa^{2}\right) .
\end{aligned}
$$

To the best of our knowledge, this fact was previously known only in special cases; see $[47,48]$.

4.1. Construction of eigenfunctions. At this point we fix the polynomials $\alpha$ and $\beta$ in (9) and the coupling constant $\kappa>0$ and consider the resulting deformed Calogero-Sutherland operator $H_{N, \tilde{N}}(\underline{6})$. As we will see, the construction of explicit series representations for the polynomials $P_{\boldsymbol{\lambda}}$ is very similar to the one in the previous section, but there are a few important differences in definitions and notation which we now specify.

We start by noting that $\Psi_{\boldsymbol{\lambda}}$ is an eigenfunction of $H_{N, \tilde{N}}$ if and only if $P_{\boldsymbol{\lambda}}$ is an eigenfunction of the differential operator

$$
\begin{aligned}
\tilde{H}_{N, \tilde{N}}:= & \Psi_{0}^{-1}\left(H_{N, \tilde{N}}-E_{0}\right) \Psi_{0}=-\sum_{j=1}^{N}\left(\alpha\left(z_{j}\right) \partial_{z_{j}}^{2}+\beta\left(z_{j}\right) \partial_{z_{j}}\right) \\
+ & \kappa \sum_{J=1}^{\tilde{N}}\left(\alpha\left(\tilde{z}_{J}\right) \partial_{\tilde{z}_{J}}^{2}+\beta_{-1 / \kappa}\left(\tilde{z}_{J}\right) \partial_{\tilde{z}_{J}}\right)-2 \kappa \sum_{j \neq k} \frac{\alpha\left(z_{k}\right)}{z_{j}-z_{k}} \partial_{z_{j}} \\
& \quad+2 \sum_{J \neq K} \frac{\alpha\left(\tilde{z}_{K}\right)}{\tilde{z}_{J}-\tilde{z}_{K}} \partial_{\tilde{z}_{J}}-2 \kappa \sum_{J, k} \frac{\alpha\left(z_{k}\right)}{\tilde{z}_{J}-z_{k}} \partial_{\tilde{z}_{J}}-2 \sum_{j, K} \frac{\alpha\left(\tilde{z}_{K}\right)}{z_{j}-\tilde{z}_{K}} \partial_{z_{j}}
\end{aligned}
$$

with corresponding eigenvalue $E_{\boldsymbol{\lambda}}-E_{0}$; this equivalence follows from a straightforward computations using that $\Psi_{0}$ (41) is an eigenstate of $H_{N, \tilde{N}}(\underline{6}$ ) with eigenvalue $E_{0}$ (Corollary 2.3 for $M=\tilde{M}=0$ ) and the identities (8), (13), and (11). We shall refer to the polynomials $P_{\boldsymbol{\lambda}}$ as reduced eigenfunctions of the deformed CalogeroSutherland operators (6). Furthermore, we let

$$
\begin{array}{r}
\tilde{F}_{N, \tilde{N}, M, \tilde{M}}(\mathbf{z}, \tilde{\mathbf{z}}, \mathbf{w}, \tilde{\mathbf{w}}) \\
=\frac{\prod_{j<k}\left(1-w_{j} / w_{k}\right)^{\kappa} \prod_{J<K}\left(1-\tilde{w}_{J} / \tilde{w}_{K}\right)^{1 / \kappa}}{\prod_{j, J}\left(1-w_{j} / \tilde{w}_{J}\right)} \\
\times \frac{\prod_{j, K}\left(1-z_{j} / \tilde{w}_{K}\right) \prod_{J, k}\left(1-\tilde{z}_{J} / w_{k}\right)}{\prod_{j, k}\left(1-z_{j} / w_{k}\right)^{\kappa} \prod_{J, K}\left(1-\tilde{z}_{J} / \tilde{w}_{K}\right)^{1 / \kappa}}
\end{array}
$$

where

$$
(\mathbf{z}, \tilde{\mathbf{z}})=\left(z_{1}, \ldots, z_{N}, \tilde{z}_{1}, \ldots, \tilde{z}_{\tilde{N}}\right) \text { and }(\mathbf{w}, \tilde{\mathbf{w}})=\left(w_{1}, \ldots, w_{N}, \tilde{w}_{1}, \ldots, \tilde{w}_{\tilde{M}}\right)
$$

and define the polynomials $f_{\mathbf{n}}^{(M, \tilde{M})}(\mathbf{z}, \tilde{\mathbf{z}}), \mathbf{n} \in \mathbb{Z}^{M+\tilde{M}}$, through the following expansion:

$$
\begin{aligned}
& \tilde{F}_{N, \tilde{N}, M, \tilde{M}}(\mathbf{z}, \tilde{\mathbf{z}}, \mathbf{w}, \tilde{\mathbf{w}}) \\
& \quad=\sum_{\mathbf{n} \in \mathbb{Z}^{M+\tilde{M}}} f_{\mathbf{n}}^{(M, \tilde{M})}(\mathbf{z}, \tilde{\mathbf{z}}) w_{1}^{-n_{1}} \cdots w_{M}^{-n_{M}} \tilde{w}_{1}^{-n_{M+1}} \cdots \tilde{w}_{\tilde{M}}^{-n_{M+\tilde{M}}}
\end{aligned}
$$


valid in the region $\left|w_{1}\right|>\cdots>\left|w_{M}\right|>\left|\tilde{w}_{1}\right|>\cdots>\left|\tilde{w}_{\tilde{M}}\right|>\max _{j, J}\left(\left|z_{j}\right|,\left|\tilde{z}_{J}\right|\right)$. The next step is to compute the action of the differential operator $\tilde{H}_{N, \tilde{N}}$ on these polynomials $f_{\mathbf{n}}^{(M, \tilde{M})}$. In order to simplify such a computation it is useful to introduce the following 'parity' function $q$ on the index set $\{1, \ldots, M+\tilde{M}\}$ :

$$
q(j):= \begin{cases}0, & \text { if } j=1, \ldots, M \\ 1, & \text { if } j=M+1, \ldots, \tilde{M}\end{cases}
$$

We also find it convenient to define the following shifted integer vectors $\mathbf{n}^{+}$associated with each quantum number $\mathbf{n}$ :

$$
n_{j}^{+}= \begin{cases}n_{j}+\kappa(N+1-j)-\tilde{N}, & \text { if } j=1, \ldots, M, \\ n_{j}+(\tilde{N}+M+1-j) / \kappa-N+M, & \text { if } j=M+1, \ldots, \tilde{M}\end{cases}
$$

Proceeding in analogy with the proof of Lemma 3.1 in the previous section it is now straightforward to obtain the following:

Lemma 4.1. For each $\mathbf{n} \in \mathbb{Z}^{M+\tilde{M}}$,

$$
\begin{gathered}
-\sum_{j=1}^{M+\tilde{M}}\left((-\kappa)^{q(j)} \alpha_{1} n_{j}^{+}\left(n_{j}^{+}-1\right)+\left(\beta_{0}-\left(1-(-\kappa)^{1-q(j)}\right) \alpha_{1}\right)\left(n_{j}^{+}-1\right)\right) f_{\mathbf{n}-\mathbf{e}_{j}}^{(M, \tilde{M})} \\
-\alpha_{0} \sum_{j=1}^{M+\tilde{M}}(-\kappa)^{q(j)}\left(n_{j}^{+}-1\right)\left(n_{j}^{+}-2\right) f_{\mathbf{n}-2 \mathbf{e}_{j}}^{(M, \tilde{M})} \\
+\sum_{j<k}(1-\kappa)(-\kappa)^{1-q(j)-q(k)} \sum_{p=0}^{2} \sum_{\nu=1}^{\infty} \alpha_{p}(2 \nu-p) f_{\mathbf{n}-\mathbf{E}_{j, k}^{p, \nu}}^{(M, \tilde{M})}
\end{gathered}
$$

with

$$
\begin{aligned}
& E_{\mathbf{n}}^{(M, \tilde{M})}-E_{0}=-\sum_{j=1}^{M}\left(\alpha_{2} n_{j}\left(n_{j}-1\right)+\left(2 \alpha_{2}(\kappa(N-j)-\tilde{N})+\beta_{1}\right) n_{j}\right) \\
& +\sum_{j=M+1}^{M+\tilde{M}}\left(\kappa \alpha_{2} n_{j}\left(n_{j}+1\right)+\left(2 \alpha_{2}(\tilde{N}-M+1-j-\kappa(N-M))-\beta_{1}\right) n_{j}\right) .
\end{aligned}
$$

Similarly to the previous section, Lemma 4.1 implies that that there exist an eigenfunction of the form

$$
P_{\mathbf{n}}^{(M, \tilde{M})}=f_{\mathbf{n}}^{(M, \tilde{M})}+\sum_{\mathbf{m}} u_{\mathbf{n}}(\mathbf{m}) f_{\mathbf{m}}^{(M, \tilde{M})}
$$


where the sum is over integer vectors $\mathbf{m} \in \mathbb{Z}^{M+\tilde{M}}$ such that $\mathbf{m} \prec \mathbf{n}$, if and only if the coefficients $u_{\mathbf{n}}(\mathbf{m})$ satisfy the recursion relation

$$
\begin{aligned}
& \left(E_{\mathbf{n}}^{(M, \tilde{M})}-E_{\mathbf{m}}^{(M, \tilde{M})}\right) u_{\mathbf{n}}(\mathbf{m}) \\
& =-\sum_{j=1}^{M+\tilde{M}} m_{j}^{+}\left((-\kappa)^{q(j)} \alpha_{1}\left(n_{j}^{+}+1\right)+\left(\beta_{0}-\left(1-(-\kappa)^{1-q(j)}\right) \alpha_{1}\right)\right) u_{\mathbf{n}}\left(\mathbf{m}+e_{j}\right) \\
& \quad-\alpha_{0} \sum_{j=1}^{M+\tilde{M}}(-\kappa)^{q(j)} m_{j}^{+}\left(m_{j}^{+}+1\right) u_{\mathbf{n}}\left(\mathbf{m}+2 e_{j}\right) \\
& \quad+(1-\kappa) \sum_{j<k}(-\kappa)^{1-q(j)-q(k)} \sum_{p=0}^{2} \sum_{\nu=1}^{\infty} \alpha_{p}(2 \nu-p) u_{\mathbf{n}}\left(\mathbf{n}+\mathbf{E}_{j, k}^{p, \nu}\right)
\end{aligned}
$$

Provided that $E_{\mathbf{n}}^{(M, \tilde{M})}-E_{\mathbf{m}}^{(M, \tilde{M})} \neq 0$ for all integer vectors $\mathbf{m}$ which appear in (51) this uniquely determines all coefficients $u_{\mathbf{n}}(\mathbf{m})$. As previously mentioned, an important feature of our construction is that the non-negative integers $M$ and $\tilde{M}$ can be chosen freely, and for each choice we obtain a family of reduced eigenfunctions $P_{\mathbf{n}}^{(M, \tilde{M})}$ of $H_{N, \tilde{N}}$ which are labelled by integer vectors $\mathbf{n} \in \mathbb{Z}^{M+\tilde{M}}$. This yields eigenfunctions (40) of $H_{N, \tilde{N}}$ with corresponding eigenvalues $E_{\mathbf{n}}^{(M, \tilde{M})}$ given by (50) and (43). A priori, it is not clear whether these eigenvalues coincide with the ones given in (42). In particular, it seems that the eigenvalues we obtain depend on the specific values of $M$ and $\tilde{M}$, but, of course, the spectrum of $H_{N, \tilde{N}}$ does not. This apparent paradox can be resolved by exhibiting an explicit mapping from the eigenvalues $E_{\mathbf{n}}^{(M, \tilde{M})}$ (50) to $E_{\boldsymbol{\lambda}}$ (42) under certain restrictions on the integer vectors $\mathbf{n} \in \mathbb{Z}^{M+\tilde{M}}$. To state this result we need the notion of the conjugate $\boldsymbol{\mu}^{\prime}$ of a partition $\boldsymbol{\mu}$, obtained by interchanging rows and columns in the Young diagram corresponding to $\boldsymbol{\mu}$, e.g.,

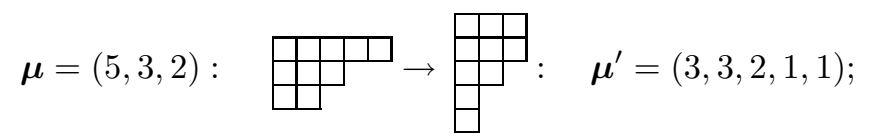

see, e.g., Section I.1 in [39].

Lemma 4.2. Let $\mathbf{n}=(\mathbf{m}, \boldsymbol{\mu})$ with $\mathbf{m} \in \mathbb{Z}^{M}$ and $\boldsymbol{\mu}$ a partition of length $\ell(\boldsymbol{\mu}) \leq \tilde{M}$. Then

$$
E_{\mathbf{n}}^{(M, \tilde{M})}=E_{\boldsymbol{\lambda}}
$$

with $\boldsymbol{\lambda}=\left(\mathbf{m}, \boldsymbol{\mu}^{\prime}\right)$.

Proof. We recall the following two well-known identities obeyed by all partitions $\boldsymbol{\mu}$ :

$$
\sum_{j} \mu_{j}^{2}=\sum_{j}(2 j-1) \mu_{j}^{\prime}, \quad \sum_{j} j \mu_{j}=\sum_{j} \frac{1}{2} \mu_{j}^{\prime}\left(\mu_{j}^{\prime}+1\right),
$$

where the sums are over all non-zero parts $\mu_{j}$ and $\mu_{j}^{\prime}$ of $\boldsymbol{\mu}$ and $\boldsymbol{\mu}^{\prime}$, respectively. This, together with the identity $\sum_{j} \mu_{j}=\sum_{j} \mu_{j}^{\prime}$ inserted into the second sum in 
(50), yields

$$
\begin{aligned}
E_{\mathbf{n}}^{(M, \tilde{M})}=E_{0}-\sum_{j=1}^{M} & \left(\alpha_{2} m_{j}\left(m_{j}-1\right)+\left(2 \alpha_{2}(\kappa(N-j)-\tilde{N})+\beta_{1}\right) m_{j}\right) \\
& -\sum_{j=1}^{\ell\left(\boldsymbol{\mu}^{\prime}\right)}\left(\alpha_{2} \mu_{j}^{\prime}\left(\mu_{j}^{\prime}-1\right)+\left(2 \alpha_{2}(\kappa(N-M-j)-\tilde{N})+\beta_{1}\right) \mu_{j}^{\prime}\right) .
\end{aligned}
$$

The statement now follows by substituting $\lambda_{j}$ for $m_{j}$ and $\lambda_{M+j}$ for $\mu_{j}^{\prime}$.

4.2. On the complexity of the series representations. The construction described in the previous section gives reduced eigenfunctions $P_{\mathbf{n}}$ for all integer vectors $\mathbf{n}$ such that a certain condition of non-degenracy on the corresponding eigenvalues is satisfied, but unless $\mathbf{n}$ satisfies the condition in (52) for some partition $\boldsymbol{\lambda}$ in the fat $(N, \tilde{N})$-hook this function ought to vanish identically. Lemma 4.2 gives, for each partition $\boldsymbol{\lambda}$ in the fat $(N, \tilde{N})$-hook, such an integer vector $\mathbf{n}$ provided one chooses $(M, \tilde{M})=(N, \tilde{N})$, and the latter choice thus is natural if one is interested in a general formula which works for (essentially) all eigenfunctions. However, we stress that we have constructed a family of reduced eigenfunctions for each choice of nonnegative integers $M$ and $\tilde{M}$, and that there exist a simple relation between any two such families: For any two sets of non-negative integers $\left(M_{1}, \tilde{M}_{1}\right)$ and $\left(M_{2}, \tilde{M}_{2}\right)$ one can show that two reduced eigenfunctions $P_{\mathbf{n}_{1}}^{\left(M_{1}, \tilde{M}_{1}\right)}$ and $P_{\mathbf{n}_{2}}^{\left(M_{2}, \tilde{M}_{2}\right)}$, labelled by integer vectors $\mathbf{n}_{1}$ and $\mathbf{n}_{2}$ which correspond to one and the same partition $\boldsymbol{\lambda}$ under the mapping defined in Lemma 4.2, are equal up to normalisation; see the first arXiv version (v1) of [18]. We can thus, in a simple manner, obtain different series representations for one and the same reduced eigenfunction by varying the values of $M$ and $\tilde{M}$. Since the complexity of this series representation is highly dependent on the values of $M$ and $\tilde{M}$, one can choose the latter such that the complexity is minimised, in many cases substantially below that of the canonical choice $(M, \tilde{M})=(N, \tilde{N})$. This is already evident in the case of the Schrödinger operators (3), i.e., for $\tilde{N}=0$. For example, suppose that we are interested in the reduced eigenfunction of the standard Sutherland model for $N=8$ and corresponding to the partition with diagram

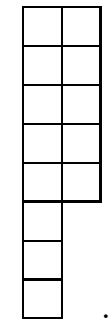

Rather than setting $M=8$ and $\tilde{M}=0$ we can use the fact that the conjugate of this partition is given by the diagram

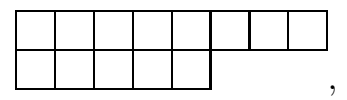

and thus the sought after reduced eigenfunction can equally well be obtained by setting $M=0$ and $\tilde{M}=2$, in the process decreasing the complexity of its series representation from the 8-particle case to that of only 2-particles. As a further 
illustrative example we consider the reduced eigenfunction corresponding to the partition with diagram

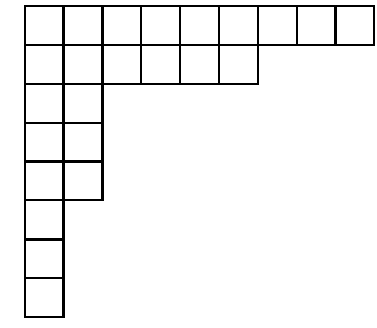

for which we can set $M=\tilde{M}=2$ to minimise the complexity of its series representation. Similar examples can be easily constructed also in the 'deformed' case. In generally, it can be readily verified that, given a specific partition $\boldsymbol{\lambda}$, the minimal value for $M+\tilde{M}$ is attained by setting $M=j$ and $\tilde{M}=\lambda_{j+1}$ where $j$ denotes the row in the diagram of $\boldsymbol{\lambda}$ for which $j+\lambda_{j+1}$ is minimal. This means, in particular, that we always can choose $M$ and $\tilde{M}$ such that their sum does not exceed the length $\ell(\boldsymbol{\lambda})$ of the partition $\boldsymbol{\lambda}$ in question, which may be less than $N+\tilde{N}$. This observation is also reflected in the fact that $f_{\mathbf{n}}^{(M, \tilde{M})}=f_{\mathbf{n}}^{(M, \tilde{M}+K)}$ for all non-negative values of $K$ as long as $M+\tilde{M} \geq \ell(\mathbf{n})$; the latter is readily inferred from the definition of the polynomials $f_{\mathbf{n}}^{(M, \tilde{M})}$.

4.3. Elementary properties of the reduced eigenfunctions. We conclude this section by verifying that the functions $f_{\mathbf{n}}^{(M, \tilde{M})}$, and consequently also the reduced eigenfunctions $P_{\mathbf{n}}^{(M, \tilde{M})}$, are contained in the algebra $\Lambda_{N, \tilde{N}, \kappa}$ for all values of the non-negative integers $M$ and $\tilde{M}$.

Lemma 4.3. Let $\mathbf{n} \in \mathbb{Z}^{M+\tilde{M}}$. Then the function $f_{\mathbf{n}}^{(M, \tilde{M})}$ is non-zero only if $\mathbf{n} \succeq 0$, and in that case it is a homogeneous polynomial of degree $|\mathbf{n}|$ in $\Lambda_{N, \tilde{N}, \kappa}$.

Proof. The proof is by straightforward computations: expanding each factor on the r.h.s. in (45) in the region $\left|w_{1}\right|>\cdots>\left|w_{M+\tilde{M}}\right|>\max _{j, J}\left(\left|z_{j}\right|,\left|\tilde{z}_{J}\right|\right)$ (recall that $\left.\tilde{w}_{J}=w_{M+J}\right)$ in a binomial series one obtains a well-defined series representation of $\tilde{F}_{N, \tilde{N}, M, \tilde{M}}$ as a linear superposition of monomials $\mathbf{w}^{-\mathbf{n}}$. By inspection one can check that in this series only terms $\mathbf{w}^{-\mathbf{n}}$ with $\mathbf{n} \succeq 0$ appear, that each such term appear only a finite number of times, and that the coefficients of $\mathbf{w}^{-\mathbf{n}}$ is a homogeneous polynomials of degree $|\mathbf{n}|$ in the variables $z_{j}$ and $\tilde{z}_{J}$. It thus follows from (46) that $f_{\mathbf{n}}^{(M, \tilde{M})}$ is non-zero only if $\mathbf{n} \succeq 0$, and in that case it is a homogeneous polynomial of degree $|\mathbf{n}|$. To prove that the $f_{\mathbf{n}}^{(M, \tilde{M})}$ are contained in $\Lambda_{N, \tilde{N}, \kappa}$ we observe that $\tilde{F}_{N, \tilde{N}, M, \tilde{M}}$ is separately symmetric in the variables $z_{j}$ and $\tilde{z}_{J}$, and that

$$
\left.\left(\partial_{z_{j}}+\kappa \partial_{\tilde{z}_{J}}\right) \tilde{F}_{N, \tilde{N}, M, \tilde{M}}\right|_{z_{j}=\tilde{z}_{J}}=0
$$

for all $j=1, \ldots, N$ and $J=1, \ldots, \tilde{N}$. The latter follows from (45) by straightforward computations,

$$
\partial_{z_{j}} \log \tilde{F}_{N, \tilde{N}, M, \tilde{M}}=\sum_{K=1}^{\tilde{M}} \frac{1}{z_{j}-\tilde{w}_{K}}-\kappa \sum_{k=1}^{M} \frac{1}{z_{j}-w_{k}}
$$


and

$$
\partial_{\tilde{z}_{J}} \log \tilde{F}_{N, \tilde{N}, M, \tilde{M}}=\sum_{k=1}^{M} \frac{1}{\tilde{z}_{J}-w_{k}}-\frac{1}{\kappa} \sum_{K=1}^{\tilde{M}} \frac{1}{\tilde{z}_{J}-\tilde{w}_{K}} .
$$

\section{CONCluding REMARKS}

In this final section we briefly discuss the relation of our construction to the so called generalised classical orthogonal polynomials of Lassalle and Macdonald, present some further remarks of the effect of spectral degeneracies on our constructions of reduced eigenfunctions of the Schrödinger operators (31) and the deformed Calogero-Sutherland operators (6), and comment on the question of completeness in the case of the deformed Calogero-Sutherland operators.

5.1. The relation to generalised classical orthogonal polynomials. As is well known, the spectra of the Schrödinger operators (3) are degenerate. In particular, in cases I and IV the eigenvalue of a given reduced eigenfunction $P_{\mathbf{n}}$ depends only on the weight $|\mathbf{n}|$ of the integer vector $\mathbf{n}$. An interesting question is thus what the relation is between the basises for the reduced eigenfunctions we construct and previously known such basises. Below we will sketch a proof of the fact that our basis coincide, up to normalisation, with the generalised classical orthogonal polynomials of Lassalle [33-35] and Macdonald [38]. We recall that these latter polynomials are reduced eigenfunctions of the Schrödinger operator in case I (Hermite), case IV (Laguerre) or case VI (Jacobi), and of the form

$$
\mathcal{P}_{\boldsymbol{\lambda}}=\sum_{\mu \subseteq \lambda} u_{\boldsymbol{\lambda} \mu} \mathcal{J}_{\boldsymbol{\mu}}
$$

for some coefficients $u_{\boldsymbol{\lambda} \boldsymbol{\mu}}$, and where $\boldsymbol{\mu} \subseteq \boldsymbol{\lambda}$ means that the Young diagram of $\boldsymbol{\mu}$ is contained in that of $\boldsymbol{\lambda}$. We shall require the following partial order on the set of partitions:

$$
\boldsymbol{\mu} \leq \boldsymbol{\lambda} \Leftrightarrow \mu_{1}+\cdots+\mu_{j} \leq \lambda_{1}+\cdots+\lambda_{j}, \quad \forall j
$$

When restricted to the set of partitions of a fixed weight this coincides with the so called dominance order. It is a simple exercise to verify that if $|\boldsymbol{\mu}|=|\boldsymbol{\lambda}|$ and $\boldsymbol{\mu} \leq \boldsymbol{\lambda}$ then $\boldsymbol{\mu} \succeq \boldsymbol{\lambda}$ and vice versa. Furthermore, comparing the expansions (27) and (37), and using the triangular structure of the Jack polynomials when expanded in monomial symmetric polynomials, one finds that

$$
g_{\boldsymbol{\lambda}}=\sum_{|\boldsymbol{\mu}|=|\boldsymbol{\lambda}|, \boldsymbol{\mu} \geq \boldsymbol{\lambda}} a_{\boldsymbol{\lambda} \boldsymbol{\mu}} \mathcal{J}_{\boldsymbol{\mu}}
$$

for some coefficients $a_{\boldsymbol{\lambda} \boldsymbol{\mu}}$ such that $a_{\boldsymbol{\lambda} \boldsymbol{\lambda}}=b_{\boldsymbol{\lambda}}$; see Equation 10.16 in Section VI.10 of [39] for the definition of $b_{\boldsymbol{\lambda}}$ (in doing so observe that the parameter $\alpha=1 / \kappa$ ). It is now readily inferred from Lemma 3.2 that any reduced eigenfunction $P_{\boldsymbol{\lambda}}$, as constructed in Section 3.1, is of the form

$$
P_{\boldsymbol{\lambda}}=\sum_{\mu \preceq \lambda} v_{\lambda \mu} \mathcal{J}_{\mu}
$$

for some coefficients $v_{\boldsymbol{\lambda} \boldsymbol{\mu}}$ such that $v_{\boldsymbol{\lambda} \boldsymbol{\lambda}}=b_{\boldsymbol{\lambda}}$. Since $\boldsymbol{\mu} \preceq \boldsymbol{\lambda}$ if $\boldsymbol{\mu} \subseteq \boldsymbol{\lambda}$ and, up to normalisation, there can be only one eigenfunction of this form, it follows that the 
relation between the reduced eigenfunctions we construct and those of Lassalle and Macdonald is given by

$$
P_{\boldsymbol{\lambda}}=b_{\boldsymbol{\lambda}} \mathcal{P}_{\boldsymbol{\lambda}} .
$$

5.2. Spectral degeneracies. We stress once more the issue of degeneracies of the eigenvalues of the Schrödinger operators (3) and the deformed Calogero-Sutherland operators (6): since our construction of reduced eigenfunction of these operators only is valid under a certain non-degeneracy condition on the corresponding eigenvalues, this issue plays a decisive role in our construction. From our point of view, it is essentially only this issue which truly distinguishes the different special cases of our results listed in Table 1. It thus would be interesting to investigate this issue further.

5.3. Completeness for deformed Calogero-Sutherland operators. We mention that one can prove an analogue of Proposition 3.2 for our construction of reduced eigenfunctions of the deformed Calogero-Sutherland operators (6). In fact, proceeding similarly to Section 3.3 one can prove that the reduced eigenfunctions constructed in Section 4.1 have a triangular expansion in so-called super Jack polynomials. We recall that the super Jack polynomials are known to constitute a linear basis for the algebra $\Lambda_{N, \tilde{N}, \kappa}$. Using these two facts above one can show that if $M \geq N$ and $\tilde{M} \geq \tilde{N}$ then the reduced eigenfunctions constructed in Section 4.1 are also a linear basis for $\Lambda_{N, \tilde{N}, \kappa}$. If either $M<N$ or $\tilde{M}<\tilde{N}$ then they span only a subspace of $\Lambda_{N, \tilde{N}, \kappa}$ which, however, can be given a rather simple characterisation. A detailed discussion of these facts can be found in the first arXiv version (v1) of [18].

\section{ACKNOWLEDGEMENTS}

We are grateful to F. Calogero, O. Chalykh, A. Fordy, P. Forrester, Y. Suris, and A. Veselov, for useful discussions. M.H. would also like to thank P. Forrester for making available to him an unpublished manuscript by Macdonald [38]. We are also grateful to two anonymous referees for constructive criticism which helped us to improve the paper. This work was significantly influenced by discussions with V. Kuznetsov. His experience, critical remarks, and interest were very valuable to us and are now sadly missed. This work was supported by the Swedish Science Research Council (VR), the Göran Gustafsson Foundation, and the European Union through the FP6 Marie Curie RTN ENIGMA (Contract number MRTN-CT-2004$5652)$.

\section{REFERENCES}

[1] M. Abramovitz and I.A. Stegun (eds.), Handbook of mathematical functions, Dover Pub., 1965.

[2] H. Awata, Y. Matsuo, S. Odake and J. Shiraishi, Excited states of the CalogeroSutherland model and singular vectors of the $W_{N}$ algebra, Nucl. Phys. B 449 (1995), $347-374$.

[3] T.H. Baker and P.J. Forrester, The Calogero-Sutherland model and generalized classical polynomials, Commun. Math. Phys. 188 (1997), 175-216.

[4] K.G. Boreskov, A.V. Turbiner, J.C. Lopez Vieyra, Solvability of the Hamiltonians related to exceptional root spaces: rational case, Commun. Math. Phys. 260 (2005), $17-44$.

[5] L. Brink, T.H. Hansson and M.A. Vasiliev, Explicit solution to the $\mathrm{N}$-body Calogero problem, Phys. Lett. B 286 (1992), 109-111. 
[6] F. Calogero, Solution of the one-dimensional $N$-body problems with quadratic and/or inversely quadratic pair potentials, J. Math. Phys. 12 (1971), 419-436.

[7] O. Chalykh, M. Feigin and A. Veselov, New integrable generalizations of CalogeroMoser quantum problems, J. Math. Phys. 39 (1998), 695-703.

[8] P. Desrosiers, L. Lapointe and P. Mathieu, Explicit formulas for the generalized Hermite polynomials in superspace, J. Phys. A: Math. Gen. 37 (2004), 1251-1268.

[9] J.F. van Diejen, Confluent hypergeometric orthogonal polynomials related to the rational Calogero system with harmonic confinement, Commun. Math. Phys. 188 (1997), 467-497.

[10] J.F. van Diejen, L. Lapointe and J. Morse, Determinantal construction of orthogonal polynomials associated with root systems, Compos. Math. 140 (2004), no. 2, 255-273.

[11] C.F. Dunkl and Y. Xu, Orthogonal polynomials of several variables, Encyclopedia of mathematics and its applications, vol. 81, Cambridge university press, 2001.

[12] P.J. Forrester, Some multidimensional integrals related to many-body systems with the $1 / r^{2}$ potential, J. Phys. A: Math. Gen. 25 (1992), L607-L614.

[13] M. Gaudin, Conjugasion $\lambda \leftrightarrow \lambda^{-1}$ de l'hamiltonien de Calogero-Sutherland, Saclay Preprint SPhT/92-158, 1992.

[14] D. Gómez-Ullate, A. González-López and M.A. Rodríguez, New algebraic quantum many-body problems, J. Phys. A: Math. Gen. 33 (2000), 7305-7335.

[15] A. González-López, N. Kamran and P.J. Olver, New quasi-exactly solvable Hamiltonians in two dimensions, Commun. Math. Phys. 159 (1994), 503-537.

[16] E. Grosswald, Bessel polynomials, Lecture notes in mathematics, vol. 698, SpringerVerlag, 1978.

[17] M. Hallnäs, An explicit formula for symmetric polynomials related to the eigenfunctions of Calogero-Sutherland models, SIGMA 3 (2007), 037 (17 pages).

[18] M. Hallnäs, A basis for the polynomial eigenfunctions of deformed Calogero-MoserSutherland operators, arXiv:0712.1496.

[19] M. Hallnäs, Multivariable Bessel polynomials related to the hyperbolic Sutherland model with external Morse potential, arXiv:0807.4740

[20] M. Hallnäs and E. Langmann, Explicit formulas for the eigenfunctions of the N-body Calogero model, J. Phys. A: Math. Gen. 39 (2006), 3511-3533.

[21] G.J. Heckman and E.M. Opdam, Root systems and hypergeometric functions I, Compos. Math. 64 (1987), 329-352.

[22] V.I. Inozemtsev and D.V. Meshcheryakov, The discrete spectrum states of finitedimensional quantum systems connected with Lie algebras, Phys. Scr. 33 (1986), 99-104.

[23] C.G. Jacobi, De functionibus alternantibus..., Crelle's Journal 22 (1841), 360-371.

[24] S. Kakei, Common algebraic structures for the Calogero-Sutherland models, J. Phys. A: Math. Gen. 29 (1996), 619-624.

[25] S. Kakei, An orthogonal basis for the $B_{n}$-type Calogero model, J. Phys. A: Math. Gen. 30 (1997), 535-541.

[26] H.L. Krall and O. Frink, A new class of orthogonal polynomials: the Bessel polynomials, Trans. Amer. Math. Soc. 65 (1949), 100-115.

[27] F. Knop and S. Sahi, A recursion and a combinatorial formula for Jack polynomials, Invent. Math. 128 (1997), 9-22.

[28] V.B. Kuznetsov, V.V. Mangazeev and E.K. Sklyanin, Q-operator and factorised separation chain for Jack polynomials, Indag. Math. 14 (2003), 451-482.

[29] V.B. Kuznetsov and E.K. Sklyanin, On Bäcklund transformations for many-body systems, J. Phys. A: Math. Gen. 31 (1998), 2241-2251.

[30] E. Langmann, Algorithms to solve the Sutherland model, J. Math. Phys. 42 (2001), 4148.

[31] E. Langmann, A method to derive explicit formulas for an elliptic generalization of the Jack polynomials, Jack, Hall-Littlewood and Macdonald polynomials (V.B. Kuznetsov and S. Sahi, eds.), Contemporary mathematics, vol. 417, American mathematical society, 2006.

[32] L. Lapointe and L. Vinet, Exact operator solution of the Calogero-Sutherland model, Commun. Math. Phys. 178 (1996), no. 2, 425-452. 
[33] M. Lassalle, Polynômes de Jacobi généralisés, C. R. Acad. Sci. Paris 312 (1991), $425-428$.

[34] M. Lassalle, Polynômes de Laguerre généralisés, C. R. Acad. Sci. Paris 312 (1991), $725-728$.

[35] M. Lassalle, Polynômes de Hermite généralisés, C. R. Acad. Sci. Paris 313 (1991), $579-582$.

[36] M. Lassalle, A short proof of generalized Jacobi-Trudi expansions for Macdonald polynomials, Jack, Hall-Littlewood and Macdonald polynomials (V.B. Kuznetsov and S. Sahi, eds.), Contemporary mathematics, vol. 417, American mathematical society, 2006.

[37] M. Lassalle and M. Schlosser, Inversion of the Pieri formula for Macdonald polynomials, Adv. Math. 202 (2006), 289-325.

[38] I.G. Macdonald, Hypergeometric functions, unpublished manuscript.

[39] I.G. Macdonald, Symmetric functions and Hall polynomials, second ed., Oxford Science Publications, 1995.

[40] K. Mimachi and Y. Yamada, Singular vectors of the Virasoro algebra in terms of Jack symmetric polynomials, Commun. Math. Phys. 174 (1995), 447-455.

[41] A. Okounkov and G. Olshanski, Shifted Jack polynomials, binomial formula, and applications, Math. Res. Lett. 4 (1997), 69-78.

[42] M.A. Olshanetsky and A.M. Perelomov, Quantum integrable systems related to Lie algebras, Phys. Rept. 94 (1983), 314-404.

[43] M. Reed and B. Simon, Methods of modern mathematical physics. II. Fourier analysis, self-adjointness, Academic Press, 1975.

[44] D. Sen, A multispecies Calogero-Sutherland model, Nucl. Phys. B 479 (1996), 554574 .

[45] D. Serban, Some properties of the Calogero-Sutherland model with reflections, J. Phys. A: Math. Gen. 30 (1997), 4215-4225.

[46] A.N. Sergeev, Calogero operator and Lie superalgebras, Theor. Math. Phys. 131 (2002), 747-764.

[47] A.N. Sergeev and A. Veselov, Deformed quantum Calogero-Moser systems and Lie superalgebras, Commun. Math. Phys. 245 (2004), 249-278.

[48] A.N. Sergeev and A. Veselov, Generalized discriminants, deformed Calogero-MoserSutherland operators and super-Jack polynomials, Adv. Math. 192 (2005), 341-375.

[49] R.P. Stanley, Some combinatorial properties of Jack symmetric functions, Adv. in Math. 77 (1989), 76-115.

[50] B. Sutherland, Exact results for a quantum many-body problem in one dimension, Phys. Rev. A 4 (1971), 2019-2021.

[51] B. Sutherland, Exact results for a quantum many-body problem in one dimension. II, Phys. Rev. A 5 (1972), 1372-1376.

[52] H. Ujino and M. Wadati, Orthogonal symmetric polynomials associated with the quantum Calogero model, J. Phys. Soc. Japan 64 (1995), 2703-2706.

[53] H. Ujino and M. Wadati, Algebraic construction of the eigenstates for the second conserved operator of the quantum Calogero model, J. Phys. Soc. Japan 65 (1996), 653-656.

[54] S. Wojciechowski, The analogue of the Bäcklund transformation for integrable manybody systems, J. Phys. A: Math. Gen. 15 (1982), L653-L657.

SisSA, Via Beirut 2-4, 34151 Trieste TS, Italy

E-mail address: hallnas@sissa.it

Theoretical physics, KTH, S-106 91 Stockholm, Sweden

E-mail address: langmann@kth.se 